Bentham OPEN
CrossMark
Content list available at: www.benthamopen.com/TOORTHJ/
DOI: $10.2174 / 1874325001610010213$

REVIEW ARTICLE

\title{
Preoperative Determinants of Patient-reported Pain and Physical Function Levels Following Total Knee Arthroplasty: A Systematic Review
}

\author{
E. Lungu ${ }^{1, *}$, P-A. Vendittoli ${ }^{2,3}$ and F. Desmeules ${ }^{2,4}$ \\ ${ }^{I}$ Department of Biomedical Sciences, Faculty of Medicine, University of Montréal, Montréal, QC, Canada \\ ${ }^{2}$ Centre de recherche de l'Hôpital Maisonneuve-Rosemont, 5415 Boul. L'Assomption, Montreal, Québec, Canada, H1T \\ $2 M 4$ \\ ${ }^{3}$ Surgery Department, Maisonneuve-Rosemont Hospital, University of Montréal Affiliated Research Center, 5415 Boul. \\ L'Assomption, Montréal, Québec, Canada, H1T $2 M 4$ \\ ${ }^{4}$ School of Rehabilitation, Faculty of Medicine, University of Montréal, Montréal, QC, Canada
}

Received: August 27, 2015

Revised: January 6, 2016

Accepted: May 5, 2016

\begin{abstract}
:
Background:

A sound knowledge of the determinants of total knee arthroplasty (TKA) outcomes could help in patient selection, preparation and education. We aimed to assess the current status of the literature evaluating preoperative determinants of early and medium term patient-reported pain and disability following TKA.
\end{abstract}

\section{Method:}

A search in Medline, Pubmed, Embase and CINAHL until October 2014 was undertaken. Selection criteria included: 1- participants undergoing primary unilateral TKA with a follow-up from 6 months to 2 years, 2- validated disease-specific patient-reported outcome measures assessing pain and/or function used as outcome measure and 3-identification of preoperative determinants obtained via multivariate analyses. Risk of bias was assessed using a modified version of the Methodology checklist for prognostic studies.

\section{Results:}

Thirty-three prognostic explanatory studies were included. Mean total score of the methodological quality was $80.7 \pm 12.2 \%$. Sociodemographic and psychosocial determinants included greater socioeconomic deprivation (both studies), greater levels of depression and/or anxiety ( 7 out of 10 studies) and greater preoperative pain catastrophizing (all 3 studies). Significant clinical determinants included worse pre-operative knee related pain or disability (20 out of 22 studies), presence or greater levels of comorbidity (12 out of 23 studies), back pain ( 4 out of 5 studies) and lower general health (all 11 studies).

\section{Conclusion:}

Several significant determinants of short to medium-term pain and functional outcomes following TKA have been summarized by studies with moderate-to-high methodological quality. No conclusions can be reached regarding the strength of the associations between significant determinants and TKA results because of heterogeneity of study methodologies and results. Further high-quality research is required.

Keywords: Determinant, Functional limitation, Knee osteoarthritis, Postoperative pain, Total knee arthroplasty.

\footnotetext{
* Address correspondence to this author at the Department of Biomedical Sciences, Faculty of Medicine, Université de Montréal, CP 6128 Succursale
} Centre-Ville, Montréal, Québec, Canada, H3C 3J7; E-mail: eugen.lungu@umontreal.ca 


\section{INTRODUCTION}

Total knee arthroplasty (TKA) is a common procedure intended at treating patients with knee osteoarthritis (OA) suffering from pain and disability [1]. Its predominant success rendered it the second most common type of orthopaedic intervention [2]. This tendency will likely maintain, as projections suggest a six-fold increase in the number of primary TKAs performed in the next decades [2]. Although TKA is generally a successful intervention, leading to amelioration in pain levels and functional status, it yields suboptimal results in up to one third of patients [3 - 7]. Sound knowledge of determinants of TKA outcomes can help in patient selection, preparation and education, especially regarding possible risks and benefits of the procedure [8]. This is particularly relevant with respect to medium-term outcomes, as after a significant amelioration three to six months postoperatively, pain and physical function levels vary little subsequently until two years following surgery [9, 10]. During this time, patients are closely monitored by their surgeons, and the medical treatment and rehabilitation can be readily altered if progress is deemed unsatisfactory.

Previous systematic reviews attempted to summarize the determinants of TKA outcomes. Santaguida et al. (2008) identified older age and female gender to be associated with worse function following TKA [8]. However, their results are based on studies published until 2001. Van Jorbegen et al. (2014) focused on protective determinants of anterior knee pain following TKA, and their findings included mostly surgical factors, namely femoral components with a posterior centre of rotation, resection of Hoffa's pad, patellar rim electrocautery and preventing combined component internal rotation [11]. Vissers et al. (2012) focused their systematic review on psychosocial factors associated with TKA outcomes and identified pain catastrophizing and lower preoperative mental health as significant determinants of poor TKA outcomes [12]. Regardless of the evidence summarized by these systematic reviews, no consensus exists concerning either the identity or the strength of association between TKA determinants and poor outcomes. Consequently, there is an evident necessity of a comprehensive review encompassing the highest quality of evidence, which can be achieved by focusing on studies employing validated patient-reported outcome-measures (PROMs) of pain and function that also gauge the independent effect of determinants via multivariate analyses [13].

The purpose of this systematic review was to assess the current status of the literature evaluating the determinants of poor outcomes in terms of pain and functional levels following TKA. We also aimed to compare the determinants according to the approach of quantifying TKA results, i.e. as a measure of patients' postoperative status or of postoperative change. Finally, because some studies evaluate pain and function either separately, such as in the case of Western Ontario and McMaster Universities Osteoarthritis Index (WOMAC) pain and function subscales, or in a combined manner (total WOMAC score), we intended to parallel determinants according to this categorization.

\section{MATERIALS AND METHODS}

\section{Literature Search and Study Identification}

A search in four databases (Medline, Pubmed, Embase and CINAHL) from their respective inception dates until October 2014 was undertaken using a combination of keywords and MESH terms (see Appendix A). Manual searches of previously published reviews and reference lists from relevant articles were also conducted. Two authors independently reviewed the titles, abstracts and full texts of the articles in order to evaluate their eligibility.

\section{Study Selection}

The following selection criteria were applied:

1. Participants were primary unilateral TKA patients with $\leq 10 \%$ of the sample undergoing unicompartmental knee arthroplasty, bilateral TKA or revision TKA

2. $\geq 90 \%$ of the study sample was diagnosed with knee OA

3. Results are presented for a follow-up between 6 months and 2 years

4. The outcome measure was a disease-specific validated PROM assessing pain and/or function

5. Identification of determinants was obtained using multivariate analyses

6. Article is published in English or French

\section{Data Extraction}

A standardized form was employed to extract data. Participants' characteristics (diagnosis, type of surgery, age and 
gender proportion), number of patients, follow-up period, outcome measures, statistical methods used and statistical adjustments, as well as significant and non-significant determinants reported by each study were recorded. Each article was extracted by one of the raters and verified by another in order to reduce the risk of extraction errors.

\section{Methodological Quality Appraisal}

Two trained reviewers independently performed the appraisal of the methodological quality of the included studies and results were discussed in order to reach consensus. In case of disagreement, a third reviewer was available for mediation of differences.

Table 1. Description of the included studies.

\begin{tabular}{|c|c|c|c|c|c|c|c|c|c|c|c|}
\hline \multirow[t]{2}{*}{ Study } & \multicolumn{4}{|c|}{ Participants } & \multirow{2}{*}{$\begin{array}{c}\text { Number } \\
\text { of } \\
\text { patients }\end{array}$} & \multirow{2}{*}{$\begin{array}{c}\text { Follow-up } \\
\text { period }\end{array}$} & \multirow[b]{2}{*}{ Outcome Measure } & \multirow{2}{*}{$\begin{array}{c}\text { Statistical } \\
\text { method }\end{array}$} & \multirow[b]{2}{*}{ Statistical adjustment } & \multicolumn{2}{|l|}{$\begin{array}{ll}\text { Results } \\
\end{array}$} \\
\hline & Diagnosis & $\begin{array}{l}\text { Type of } \\
\text { Surgery }\end{array}$ & $\begin{array}{c}\text { Mean Age } \\
\text { (SD) }\end{array}$ & \begin{tabular}{|c|}
$\begin{array}{c}\text { Gender } \\
\text { (\%female) }\end{array}$ \\
\end{tabular} & & & & & & Significant Determinants & Non-significant Determinants \\
\hline \multirow[t]{3}{*}{$\begin{array}{l}\text { Alzahrani et al. } \\
\text { (2011) [33] }\end{array}$} & \multirow[t]{3}{*}{\begin{tabular}{|l|} 
Primary or \\
secondary $\mathrm{OA}$
\end{tabular}} & \multirow[t]{3}{*}{\begin{tabular}{|l|} 
Primary \\
unilateral TKA
\end{tabular}} & \begin{tabular}{|l|} 
Cohort A: \\
$67.5(9.6)$ \\
\end{tabular} & \begin{tabular}{|l|} 
Cohort A: \\
$62 \%$
\end{tabular} & \begin{tabular}{|l|} 
Cohort A: \\
457
\end{tabular} & \multirow[t]{3}{*}{1 year } & \multirow{3}{*}{\begin{tabular}{|l|} 
No clinical improvement at 1 \\
year: Minimal Clinically \\
Important Difference \\
WOMAC: $\leq 7.5 / 100$ points \\
OKS: $\leq 5.0 / 60$ points
\end{tabular}} & \multirow[t]{3}{*}{$\begin{array}{l}\text { Logistic } \\
\text { regression }\end{array}$} & \multirow{3}{*}{\begin{tabular}{|l} 
Age, \\
Gender \\
BMI \\
Comorbidity
\end{tabular}} & $\begin{array}{l}\text { Cohort A: Increased age at time of surgery (-): OR } \\
1.06(95 \% \text { CI } 1.02-1.09)\end{array}$ & \begin{tabular}{|l} 
Cohort A Gender BMI \\
Comorbidity
\end{tabular} \\
\hline & & & \begin{tabular}{|l|} 
Cohort B: \\
$69.0(9.1)$ \\
\end{tabular} & $\begin{array}{l}\text { Cohort B: } \\
63 \%\end{array}$ & $\begin{array}{l}\text { Cohort B: } \\
2720\end{array}$ & & & & & \multirow{2}{*}{$\begin{array}{l}\text { Cohort B: Male gender (+): OR } 0.72(95 \% \mathrm{CI} \\
0.57-0.92)\end{array}$} & \multirow[t]{2}{*}{ Cohort B: Age BMI Comorbidity } \\
\hline & & & \begin{tabular}{|l|} 
Overall: \\
$68.2(9.4)$
\end{tabular} & $\begin{array}{l}\text { Overall: } \\
63 \%\end{array}$ & \begin{tabular}{|l|}
$\begin{array}{l}\text { Overall: } \\
3177\end{array}$ \\
\end{tabular} & & & & & & \\
\hline \begin{tabular}{|l} 
Ayers $e t a l$ al. \\
$(2005)[42]$
\end{tabular} & $\mathrm{OA}$ & \begin{tabular}{|l|} 
Primary \\
unilateral TKA
\end{tabular} & $68.0(9.8)$ & $62.4 \%$ & 165 & 12 months & $\begin{array}{l}12 \text { month WOMAC-Physical } \\
\text { function score improvement } \\
\text { (change score) }\end{array}$ & \begin{tabular}{|l|} 
Blocked multiple \\
regression
\end{tabular} & NONE & \begin{tabular}{|l|} 
Age (unclear) \\
Gender (unclear) \\
Worse preoperative physical function (WOMAC \\
function) $(-)$ \\
Higher pre-operative mental health (SF-36 mental \\
component score) $(+)$
\end{tabular} & NONE \\
\hline $\begin{array}{l}\text { Baker } \text { et al. } \\
(2012)[16]\end{array}$ & $\mathrm{OA}$ & \begin{tabular}{|l|} 
Primary \\
unilateral TKA
\end{tabular} & $\begin{array}{l}\text { Not } \\
\text { available }\end{array}$ & $\begin{array}{l}\text { Not } \\
\text { available }\end{array}$ & 22691 & \begin{tabular}{|l|}
6 \\
-12 months \\
(median 199 \\
days)
\end{tabular} & improvement (change score) & \begin{tabular}{|l|} 
Stepwise multiple \\
linear regression
\end{tabular} & NONE & 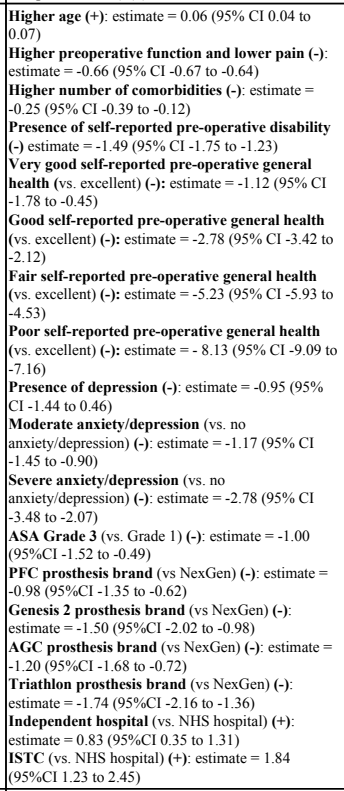 & ASA Grade 2 \\
\hline $\begin{array}{l}\text { Caracciolo } \text { et al. } \\
\text { (2005) }[37]\end{array}$ & $\mathrm{OA}$ & Primary TKA & $71.6(6.6)$ & $81 \%$ & 47 & 6 months & $\begin{array}{l}\text { WOMAC function score at } 6 \\
\text { months }\end{array}$ & \begin{tabular}{|l|} 
Logistic \\
regression
\end{tabular} & NONE & $\begin{array}{l}\text { Higher preoperative function }(+), \mathrm{OR}=1.15,95 \% \\
\mathrm{CI}=1.04 \text { to } 1.28 \text {, compared to lower preoperative } \\
\text { function (worst quartile of WOMAC function score) }\end{array}$ & \begin{tabular}{|l|}
$\begin{array}{l}\text { Preoperative osteoarthritis } \\
\text { morbidity: } \\
\text { Charnley or Modified Charnley } \\
\text { Class C }\end{array}$ \\
\end{tabular} \\
\hline \begin{tabular}{|l} 
Clement et al. \\
(2013) [32]
\end{tabular} & Primary $\mathrm{OA}$ & \begin{tabular}{|l|} 
Primary \\
unilateral TKA
\end{tabular} & $70.4(9.4)$ & 57.5 & 2392 & 1 year & 1 year OKS score & \begin{tabular}{|l|}
$\begin{array}{l}\text { Multivariate } \\
\text { linear regression } \\
\text { analysis }\end{array}$ \\
\end{tabular} & NONE & 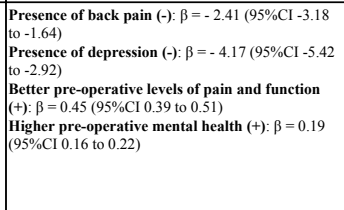 & \begin{tabular}{|l|} 
Gender \\
Heart disease \\
Hypertension \\
Lung disease \\
Vascular disease \\
Neurological disease \\
Diabetes mellitus \\
Gastric ulceration \\
Kidney disease \\
Liver disease \\
Anemia
\end{tabular} \\
\hline $\begin{array}{l}\text { Clement et al. } \\
\text { (2013) [35] }\end{array}$ & $\mathrm{N} / \mathrm{A}$ & Primary TKA & 70.4 & 56.6 & 2389 & 1 year & 1 year OKS score & \begin{tabular}{|l|} 
Multivariate \\
linear regression \\
analysis
\end{tabular} & NONE & $\begin{array}{l}\text { Vascular comorbidity (-): } \beta=-1.91,95 \% \mathrm{CI}-3.78 \\
\text { to }-0.05 \\
\text { Depression (-): } \beta=-4.19,95 \% \mathrm{CI}-5.44 \text { to }-2.95 \\
\text { Back pain (-): } \beta=-2.38,95 \% \mathrm{CI}-3.14 \text { to }-1.61 \\
\text { Better pre-0perative levels of pain and function } \\
\text { (+): } \beta=0.45,95 \% \text { C } 0.39 \text { to } 0.51 \\
\text { Higher pre-operative mental health (+): } \beta=0.19 \\
\text { (95\% } \mathrm{Cl} 0.16 \text { to } 0.22)\end{array}$ & \begin{tabular}{|l|} 
Heart disease High blood \\
pressure \\
Lung disease \\
Neurological disease \\
Diabetes \\
Stomach ulcer \\
Kidney Disease \\
Liver disease \\
Anemia \\
Pre-operative physical health
\end{tabular} \\
\hline \begin{tabular}{|l} 
Clement et al. \\
(2013) [40]
\end{tabular} & Primary OA & Primary TKA & $70.6(7.0)$ & 57.6 & 966 & 1 year & $\begin{array}{l}\text { Mean OKS improvement } \\
\text { after 1 year }\end{array}$ & \begin{tabular}{|l|}
$\begin{array}{l}\text { Multivariate } \\
\text { linear regression } \\
\text { analysis }\end{array}$ \\
\end{tabular} & NONE & \begin{tabular}{|l} 
Presence of back pain (-): $\beta=-2.53,95 \% \mathrm{CI}-3.75$ \\
-to -1.30 \\
More than 4 comorbidities (-): $\beta=-3.78,95 \% \mathrm{CI}$ \\
-6.11 to -1.45 \\
Higher preoperative function and lower pain (-): $\beta$ \\
$=0.58,95 \% \mathrm{CI} 0.50$ to 0.87 \\
Higher properative mental health (+): $\beta=0.16$, \\
$95 \% \mathrm{CI} 0.11$ to 0.22 \\
\end{tabular} & NONE \\
\hline \begin{tabular}{|l|} 
Davis et al (2008) \\
{$[34]$}
\end{tabular} & Primary OA & $\begin{array}{l}\text { Unilateral } \\
\text { primary TKA }\end{array}$ & $81.1(49$ to & 51.0 & 974 & $\begin{array}{l}12 \text { and } 24 \\
\text { months }\end{array}$ & $\begin{array}{l}12 \text { and } 24 \text { month total } \\
\text { WOMAC score and } \\
\text { WOMAC pain and function } \\
\text { scores }\end{array}$ & \begin{tabular}{|l|}
$\begin{array}{l}\text { Multivariate } \\
\text { linear regression } \\
\text { analysis }\end{array}$ \\
\end{tabular} & \begin{tabular}{|l|} 
Age, \\
Gender \\
Number of co-morbid \\
conditions \\
Country \\
Center within country \\
Pre-operative status \\
\end{tabular} & \begin{tabular}{|l|} 
WOMAC pain \\
12 months \\
Low income (-):p $=0.014$
\end{tabular} & \begin{tabular}{|l} 
WOMAC pain \\
Low income at 24 months \\
Education status at 3,12 and 24 \\
months \\
WOMAC function \\
Low income at 12 and 24 months \\
Education status at 12 and 24 \\
months \\
\end{tabular} \\
\hline
\end{tabular}




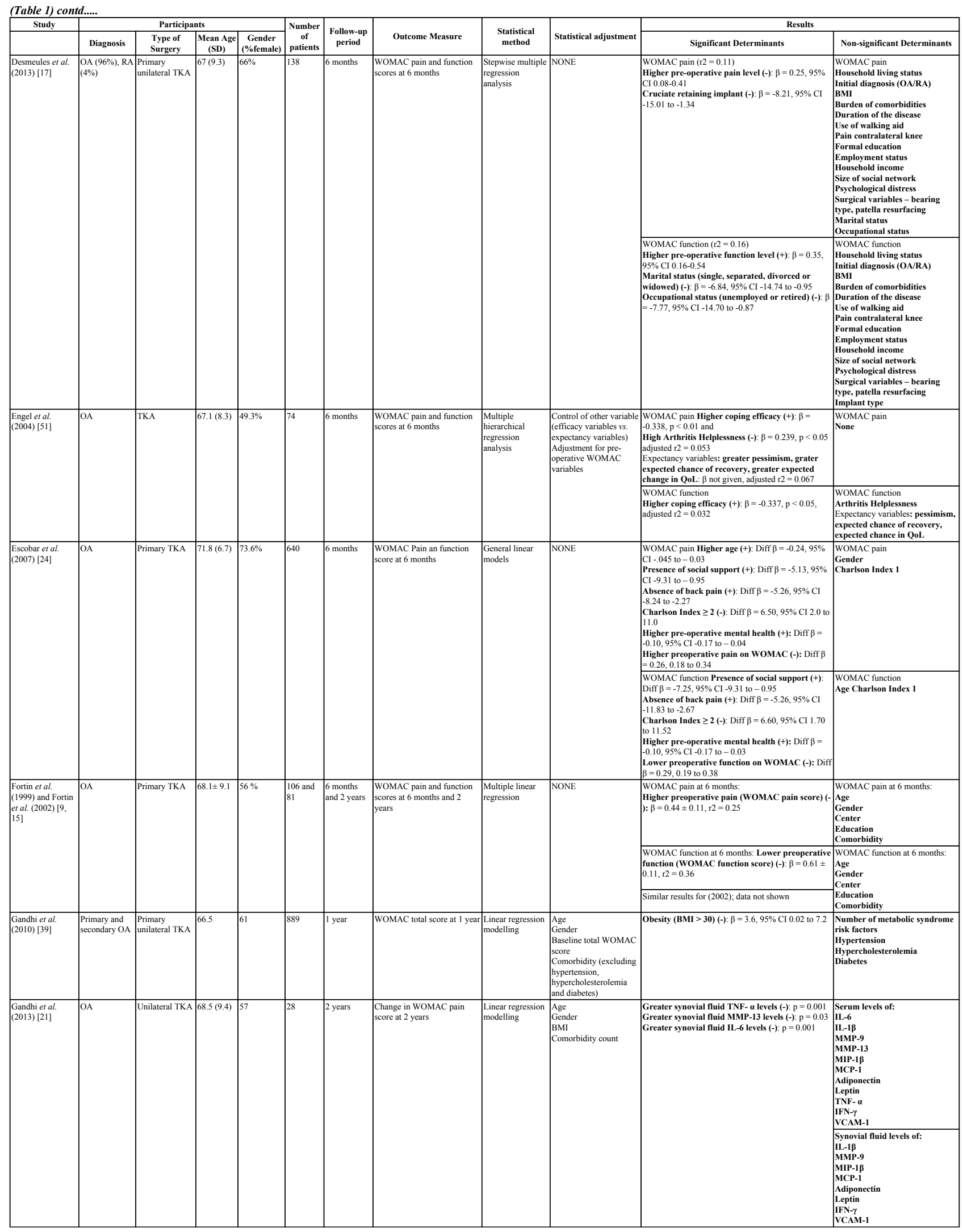




\begin{tabular}{|c|c|c|c|c|c|c|c|c|c|c|c|}
\hline \multirow[t]{2}{*}{$\begin{array}{l}\text { Study } \\
\end{array}$} & \multicolumn{4}{|c|}{ Participants } & \multirow{2}{*}{$\left|\begin{array}{c}\text { Number } \\
\text { of } \\
\text { patients }\end{array}\right|$} & \multirow{2}{*}{$\begin{array}{c}\text { Follow-up } \\
\text { period }\end{array}$} & \multirow[b]{2}{*}{ Outcome Measure } & \multirow{2}{*}{$\begin{array}{c}\text { Statistical } \\
\text { method }\end{array}$} & \multirow[b]{2}{*}{ Statistical adjustment } & \multicolumn{2}{|l|}{ Results } \\
\hline & Diagnosis & $\begin{array}{l}\text { Type of } \\
\text { Surgery }\end{array}$ & $\begin{array}{c}\text { Mean Age } \\
\text { (SD) }\end{array}$ & \begin{tabular}{|c}
$\begin{array}{c}\text { Gender } \\
\text { \%female) }\end{array}$ \\
\end{tabular} & & & & & & Significant Determinants & Non-significant Determinants \\
\hline \multirow[t]{3}{*}{\begin{tabular}{|l} 
Hanusch et al. \\
(2014) [27]
\end{tabular}} & \multirow[t]{3}{*}{$\mathrm{OA}$} & \multirow[t]{3}{*}{ Primary TKA } & \multirow[t]{3}{*}{$\begin{array}{l}71(42 \text { to } \\
92)\end{array}$} & \multirow[t]{3}{*}{45} & \multirow[t]{3}{*}{100} & \multirow[t]{3}{*}{1 year } & \multirow[t]{3}{*}{1 year OKS score } & \multirow[t]{3}{*}{\begin{tabular}{|l|}
$\begin{array}{l}\text { Stepwise multiple } \\
\text { linear regression }\end{array}$ \\
\end{tabular}} & \multirow[t]{3}{*}{ NONE } & \begin{tabular}{|l|}
$\begin{array}{l}\text { Model 1 } \\
\text { None }\end{array}$ \\
\end{tabular} & \begin{tabular}{|l|} 
Model 1 \\
Age \\
Gender \\
Pre-operative OKS score \\
Consequences (patitent's beliefs \\
about impact of illness on their \\
lie \\
Emotional representation \\
(patient's negative emotions \\
caused by their illness
\end{tabular} \\
\hline & & & & & & & & & & $\begin{array}{l}\text { Model } 2 \\
\text { Better pre-operative function and lower pain }(+) \text { : } \\
\beta=-0.296, p=0.008 \\
\text { Higher anxiety }(-): \beta=0.270, p=0.01\end{array}$ & \begin{tabular}{|l|} 
Model 2 \\
Age \\
Gender
\end{tabular} \\
\hline & & & & & & & & & & $\begin{array}{l}\text { Model } 3 \\
\text { Better pre-operative function and lower pain }(+) \text { : } \\
\beta=-0.239, p=0.04 \\
\text { Higher anxiety }(-): \beta=0.296, p=0.01\end{array}$ & \begin{tabular}{|l|} 
Model 3 \\
Age Gender
\end{tabular} \\
\hline \multirow[t]{2}{*}{\begin{tabular}{|l|}
$\begin{array}{l}\text { Jones } \text { et al. }(2001) \\
{[411]}\end{array}$ \\
\end{tabular}} & \multirow[t]{2}{*}{\begin{tabular}{|l} 
Osteoarthritis \\
$(93 \%)$
\end{tabular}} & \multirow[t]{2}{*}{$\begin{array}{l}\text { Primary } \\
\text { unilateral TKA }\end{array}$} & \multirow[t]{2}{*}{70.6} & \multirow[t]{2}{*}{59} & \multirow[t]{2}{*}{257} & \multirow[t]{2}{*}{6 months } & \multirow[t]{2}{*}{$\begin{array}{l}\text { Change in WOMAC pain and } \\
\text { function scores at } 6 \text { months }\end{array}$} & \multirow[t]{2}{*}{\begin{tabular}{|l|} 
Multiple linear \\
regression models
\end{tabular}} & \multirow[t]{2}{*}{ NONE } & $\begin{array}{l}\text { Change in pain } \\
\text { Higher preoperative bodily pain (SF-36) (-): } \beta= \\
-0.42,95 \% \text { CI }-0.56 \text { to }-0.27 \text { Cementless prosthesis } \\
(-): \beta=-9.48,95 \% \text { CI }-16.20 \text { to }-2.77\end{array}$ & \begin{tabular}{|l|} 
Change in pain Age \\
Gender \\
Waiting time \\
Number of comorbid conditions
\end{tabular} \\
\hline & & & & & & & & & & \begin{tabular}{|l|} 
Change in function \\
Lower preoperative joint pain (WOMAC) (-): $\beta=$ \\
$-0.43,95 \%$ C CI - 0.5 to to -0.28 \\
Higher number of comorbid conditions \\
(-): $\beta=-1.56,95 \%$ CI - 2.74 to -0.37 \\
Higher preperative bodily pain (SF-36) (-): $\beta=$ \\
$-0.21,95 \%$ CI -0.35 to -0.07
\end{tabular} & \begin{tabular}{|l|} 
Change in function \\
Age \\
Gender \\
Waiting time \\
BMI \\
Contralateral joint involvement \\
Living alone
\end{tabular} \\
\hline 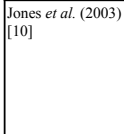 & $\mathrm{OA}(94 \%)$ & Primary TKA & $69.2(9.2)$ & 59 & 273 & 6 months & $\begin{array}{l}\text { WOMAC function score at } 6 \\
\text { months }\end{array}$ & $\begin{array}{l}\text { Multiple linear } \\
\text { regression }\end{array}$ & NONE & $\begin{array}{l}\text { Older age (+): } \beta=0.35,95 \% \mathrm{CI} 0.10 \text { to } 0.60 \\
\text { Higher preoperative function (WOMAC) (+): } \beta= \\
0.30,95 \% \mathrm{CI} 0.16 \text { to } 0.43 \\
\text { Greater number of comorbid conditions (-): } \beta= \\
-1.62,95 \% \mathrm{Cl}-2.75 \text { to }-0.49 \\
\text { Use of walking devices pre-operatively (-): } \beta= \\
-4.15,95 \% \mathrm{CI}-7.23 \text { to }-1.06\end{array}$ & Gender \\
\hline \multirow[t]{6}{*}{\begin{tabular}{|l} 
Judge et al (2012) \\
[25]
\end{tabular}} & \multirow[t]{6}{*}{$\mathrm{OA}(93.7 \%)$} & \multirow[t]{6}{*}{$\begin{array}{l}\text { Primary TKA } \\
(92 \%) \text { UKA } \\
(8 \%)\end{array}$} & \multirow[t]{6}{*}{$71.7(9.1)$} & \multirow[t]{6}{*}{61} & \multirow[t]{6}{*}{1991} & \multirow[t]{6}{*}{6 months } & \multirow[t]{3}{*}{$\begin{array}{l}\text { Model 1: } \\
\text { Total OKS, OKS pain score } \\
\text { and OKS function score at } 6 \\
\text { months }\end{array}$} & \begin{tabular}{|l|} 
Model 1: \\
Multiple linear \\
regression
\end{tabular} & $\begin{array}{l}\text { Model 1: } \\
\text { NONE }\end{array}$ & 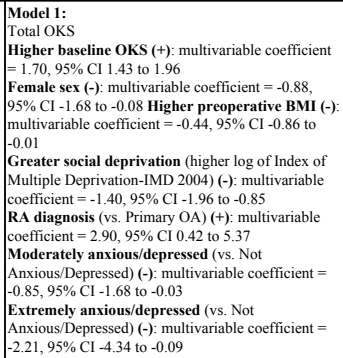 & \begin{tabular}{|l|} 
Total OKS \\
Age \\
Operated side \\
Diagnosis other than OA or RA \\
ASA grade \\
Year surgery was performed \\
\\
\end{tabular} \\
\hline & & & & & & & & & & 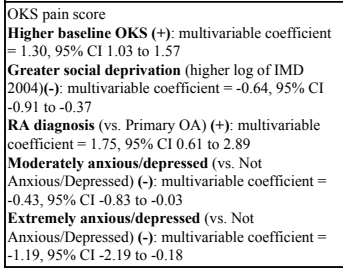 & \begin{tabular}{|l|} 
OKS pain score \\
Age \\
Gender \\
Preoperative BMI \\
Operated side \\
Diagnosis other than OA or RA \\
ASA grade \\
Year surgery was performed \\
\\
\\
\end{tabular} \\
\hline & & & & & & & & & & $\begin{array}{l}\text { OKS function score } \\
\text { Higher baseline OKS (+): multivariable coefficient } \\
=1.82,25 \% \text { CI } 1.58 \text { to } 2.06 \\
\text { Higher age (-): multivariable coefficient }=-0.21, \\
95 \% \text { CI }-0.34 \text { to }-0.08 \\
\text { Female sex (-) : multivariable coefficient }=-0.79, \\
95 \% \text { CI }-1.25 \text { to }-0.33 \\
\text { Higher preoperative BMI (-): multivariable } \\
\text { coefficient }-0.33,95 \% \text { CI - } 0.57 \text { to }-0.09 \\
\text { Greater social deprivation (higher log of IMD } \\
\text { 2004)(-): multivariable coefficient }=-0.79,95 \% \mathrm{CI} \\
-1.11 \text { to }-0.46\end{array}$ & \begin{tabular}{|l} 
OKS function score \\
Operated side \\
Diagnosis other than OA or RA \\
RA diagnosis \\
AAA grade \\
Anxiety/depression level \\
Year surgery was performed
\end{tabular} \\
\hline & & & & & & & \begin{tabular}{|l|} 
Model 2: \\
PASS score for Total OKS, \\
OKS pain score and OKS \\
function score at 6 months \\
\end{tabular} & \begin{tabular}{|l|} 
Model 2: \\
Logistic \\
regression
\end{tabular} & \begin{tabular}{|l|} 
Model 2: \\
NONE
\end{tabular} & $\begin{array}{l}\text { Model 2: } \\
\text { PASS total OKS score } \\
\text { Higher baseline OKS (+): OR }=1.52,95 \% \text { CI } 1.40 \\
\text { to } 1.66 \text { Greater social deprivation (higher log of } \\
\text { Index of Multiple Deprivation 2004) }(- \text { : OR }=0.73 \text {, } \\
95 \% \text { CI } 0.62 \text { to } 0.87 \text { RA diagnosis }(+) \text { : OR }=2.17, \\
95 \% \text { CI } 1.02 \text { to } 4.60 \\
\end{array}$ & \begin{tabular}{|l|l|} 
Model 2: \\
PASS total OKS score \\
Age \\
Gender \\
BnII \\
Operated Side \\
Diagnosis other than OA or RA \\
ASA grade \\
Anxiety/depression level \\
Year surgery was performed \\
\end{tabular} \\
\hline & & & & & & & & & & $\begin{array}{l}\text { PASS OKS pain score } \\
\text { Higher baseline OKS }(+) \text { : } \mathrm{OR}=1.81,95 \% \mathrm{CI} 1.52 \\
\text { to 2.17 } \\
\text { Greater social deprivation (higher log of Index of } \\
\text { Multiple Deprivation } 2004)(-): \mathrm{OR}=0.80,95 \% \mathrm{CI} \\
0.68 \text { to } 0.94 \\
\text { RA diagnosis (+): } \mathrm{OR}=2.33,95 \% \mathrm{Cl} 1.03 \text { to } 5.29 \\
\text { Moderately anxious/depressed (vs. not } \\
\text { anxious/depressed) }(-): \mathrm{OR}=0.67,95 \% \mathrm{CI} 0.54 \text { to } \\
0.84 \\
\text { Extremely anxious/depressed (vs. not } \\
\text { anxious/depressed) }(-): \mathrm{OR}=0.51,95 \% \mathrm{CI} 0.31 \text { to } \\
0.84\end{array}$ & \begin{tabular}{|l|} 
PASS OKS pain score \\
Age \\
Gender \\
BMI \\
Operated Side \\
Diagnosis other than OA or RA \\
AAA grade \\
Year surgery was performed \\
\end{tabular} \\
\hline & & & & & & & & & & $\begin{array}{l}\text { PASS OKS function score } \\
\text { Higher baseline OKS ( }(+) \text { : OR }=2.08,95 \% \mathrm{CI} 1.82 \\
\text { to } 2.39 \\
\text { Oder age (-): } \mathrm{OR}=0.93,95 \% \mathrm{CI} 0.87 \text { to } 0.99 \\
\text { Greater social deprivation (higher log of Index of } \\
\text { Multiple Deprivation 2004) (-): } \mathrm{OR}=0.76,95 \% \mathrm{CI} \\
0.64 \text { to } 0.89 \\
\text { Moderately anxious/depressed (vs. not } \\
\text { anxious/depressed) (-): } \mathrm{OR}=0.77,95 \% \mathrm{CI} 0.61 \text { to } \\
0.97\end{array}$ & \begin{tabular}{|l|} 
PASS OKS function score \\
Gender \\
BMI \\
Operated Side \\
Diagnosis other than OA or RA \\
ASA grade \\
Extremely anxious/depressed \\
(vs. not anxious/depressed) \\
Year surgery was performed \\
\end{tabular} \\
\hline
\end{tabular}




\begin{tabular}{|c|c|c|c|c|c|c|c|c|c|c|c|}
\hline \multirow[t]{2}{*}{ Study } & \multicolumn{4}{|c|}{ Participants } & \multirow{2}{*}{\begin{tabular}{|c}
$\begin{array}{c}\text { Number } \\
\text { of } \\
\text { patients }\end{array}$ \\
\end{tabular}} & \multirow{2}{*}{$\begin{array}{c}\text { Follow-up } \\
\text { period }\end{array}$} & \multirow[b]{2}{*}{ Outcome Measure } & \multirow[b]{2}{*}{$\begin{array}{c}\text { Statistical } \\
\text { method }\end{array}$} & \multirow[b]{2}{*}{ Statistical adjustment } & \multicolumn{2}{|l|}{ Results } \\
\hline & Diagnosis & $\begin{array}{l}\text { Type of } \\
\text { Surgery }\end{array}$ & $\begin{array}{c}\begin{array}{c}\text { Mean Age } \\
\text { (SD) }\end{array} \\
\end{array}$ & \begin{tabular}{|c|}
$\begin{array}{c}\text { Gender } \\
(\% \text { female })\end{array}$ \\
\end{tabular} & & & & & & Significant Determinants & Non-significant Determinants \\
\hline \multirow[t]{2}{*}{$\begin{array}{l}\text { Kauppila et al. } \\
\text { (2011) [4] }\end{array}$} & \multirow[t]{2}{*}{$\mathrm{OA}$} & \multirow[t]{2}{*}{ Primary TKA } & \multirow[t]{2}{*}{\begin{tabular}{|l|}
$70.7(5.5)$ \\
\end{tabular}} & \multirow[t]{2}{*}{75} & \multirow[t]{2}{*}{88} & \multirow[t]{2}{*}{12 months } & $\begin{array}{l}12 \text { month WOMAC function } \\
\text { change score }\end{array}$ & \begin{tabular}{|l} 
Multiple linear \\
regression
\end{tabular} & \multirow[t]{2}{*}{ NONE } & $\begin{array}{l}\text { Multiple linear regression } \\
\text { Male gender (-): } \beta=-12.0,95 \% \mathrm{CI}-23.1 \text { to }-0.9 \\
\text { Presence of osteoporosis (-): } \beta=-17.5,95 \% \mathrm{CI} \\
-32.9 \text { to }-2.1 \\
\text { Higher pre-operative function (-): } \beta=0.31,95 \% \mathrm{CI} \\
0.06 \text { to } 0.56\end{array}$ & $\begin{array}{l}\text { Multiple linear regression } \\
\text { Age } \\
\text { Pre-operative function of the } \\
\text { opposite knee }\end{array}$ \\
\hline & & & & & & & $\begin{array}{l}\text { OMERACT-OARSI } \\
\text { responder criteria }\end{array}$ & \begin{tabular}{|l|} 
Multivariate \\
logistic regression
\end{tabular} & & \begin{tabular}{|l|} 
Multivariate logistic regression \\
Presence of osteoporosis (-): $\mathrm{OR}=14.7,95 \% \mathrm{CI} 1.1$ \\
to 106.1
\end{tabular} & $\begin{array}{l}\text { Multivariate logistic regression } \\
\text { Data not shown }\end{array}$ \\
\hline \multirow{6}{*}{\begin{tabular}{|l} 
Lingard et al. \\
(2004) [26]
\end{tabular}} & \multirow[t]{6}{*}{ OA } & \multirow[t]{6}{*}{ Primary TKA } & \multirow[t]{6}{*}{69.9} & \multirow[t]{6}{*}{59.2} & \multirow[t]{6}{*}{860} & \multirow{6}{*}{$\begin{array}{l}12 \text { and } 24 \\
\text { months }\end{array}$} & \multirow{6}{*}{$\begin{array}{l}\text { WOMAC pain and function } \\
\text { at } 12 \text { and } 24 \text { months }\end{array}$} & \multirow{6}{*}{\begin{tabular}{|l|} 
Hierarchical \\
linear modelling
\end{tabular}} & \multirow[t]{6}{*}{ NONE } & WOMAC pain & WOMAC pain \\
\hline & & & & & & & & & & $\begin{array}{l}\text { At } 12 \text { months } \\
\text { Female gender (-): } \mathrm{F}=7.06, \mathrm{p}<0.05 \text {, parameter } \\
\text { estimate }=-3.77,95 \% \text { CI }-6.55 \text { to }-0.99 \\
\text { Lower preoperative pain (WOMAC pain score) (- }(- \\
: \mathrm{F}=29.16, \mathrm{p}<0.0005 \text {, parameter estimate }=0.20 \text {, } \\
95 \% \mathrm{CI} 0.13 \text { to } 0.28 \\
\text { Lower preoperative mental health (SF-36 mental } \\
\text { health seore) (-): } \mathrm{F}=17.53, \mathrm{p}<0.0005, \text { parameter } \\
\text { estimate }=0.16,95 \% \mathrm{CI} 0.09 \text { to } 0.24 \\
\text { More comorbid conditions (-): } \mathrm{F}=5.85, \mathrm{p}<0.05 \text {, } \\
\text { parameter estimate }=-1.33,95 \% \mathrm{CI}-2.41 \text { to }-0.25\end{array}$ & \begin{tabular}{|l} 
At 12 months \\
Age \\
Country \\
\\
\end{tabular} \\
\hline & & & & & & & & & & 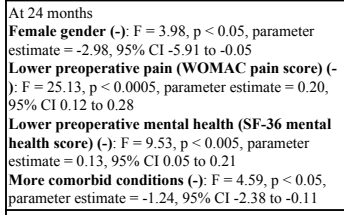 & \begin{tabular}{|l|l|} 
At 24 months \\
Age \\
\\
\end{tabular} \\
\hline & & & & & & & & & & \begin{tabular}{|l|} 
WOMAC function \\
\end{tabular} & WOMAC function \\
\hline & & & & & & & & & & 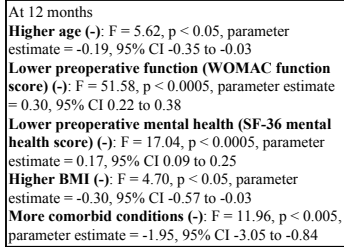 & \begin{tabular}{|l|} 
At 12 months \\
Gender
\end{tabular} \\
\hline & & & & & & & & & & 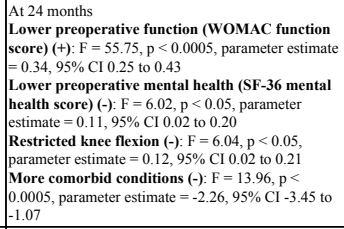 & 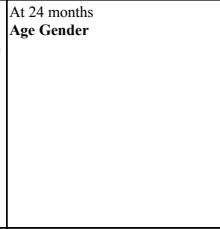 \\
\hline \begin{tabular}{|l} 
Lingard et al. \\
(2004) [26]
\end{tabular} & $\mathrm{OA}$ & Primary TKA & 69.9 & 59.2 & 860 & $\begin{array}{l}12 \text { and } 24 \\
\text { months }\end{array}$ & $\begin{array}{l}\text { WOMAC pain and function } \\
\text { at } 12 \text { and } 24 \text { months }\end{array}$ & $\begin{array}{l}\text { Hierarchical } \\
\text { linear modelling }\end{array}$ & NONE & $\begin{array}{l}\text { Lower preoperative function (WOMAC function } \\
\text { score) }(+) ; \mathrm{F}=55.75, \mathrm{p}<0.0005 \text {, parameter estimate } \\
=0.34,95 \% \mathrm{CI} 0.25 \text { to } 0.43 \\
\text { Lower preoperative mental health (SF-36 mental } \\
\text { health score) }(-) \mathrm{F}=6.02, \mathrm{p}<0.05 \text {, parameter } \\
\text { estimate }=0.11,95 \% \mathrm{CI} 0.02 \text { to } 0.20 \\
\text { Restricted knee flexion }(-) \mathrm{F}=6.04, \mathrm{p}<0.05, \\
\text { parameter estimate }=0.12,95 \% \mathrm{CI} 0.02 \text { to } 0.21 \\
\text { More comorbid conditions }(-) \mathrm{F}=13.96, \mathrm{p}< \\
0.0005 \text {, parameter estimate }=-2.26,95 \% \mathrm{CI}-3.45 \text { to } \\
-1.07\end{array}$ & \\
\hline $\begin{array}{l}\text { Lingard et al. } \\
\text { (2007) [38] }\end{array}$ & $\mathrm{OA}$ & Primary TKA & 70.8 & 60.3 & 952 & $\begin{array}{l}12 \text { and } 24 \\
\text { months }\end{array}$ & $\begin{array}{l}\text { WOMAC pain and function } \\
\text { at } 12 \text { and } 24 \text { months }\end{array}$ & $\begin{array}{l}\begin{array}{l}\text { General linear } \\
\text { models }\end{array} \\
\end{array}$ & \begin{tabular}{|l|} 
Age \\
Gender \\
Number of \\
comorbidities \\
Country \\
Center within country \\
Preoperative scores
\end{tabular} & \begin{tabular}{|l} 
With substitution of missing values \\
WOMAC pain Higher preoperative mental health \\
(SF-36) (+): \\
At 12 months: parameter estimate $=0.128, \mathrm{p}=$ \\
0.0008 \\
At 24 months: parameter estimate $=0.096, \mathrm{p}=$ \\
0.0109 \\
WOMAC function \\
Higher preoperative mental health $(\mathrm{SS}-36)(+)$ : \\
At 12 months: parameter estimate $=0.150, \mathrm{p}=$ \\
0.0001 \\
At 24 months: parameter estimate $=0.106, \mathrm{p}=$ \\
0.0071 \\
\end{tabular} & \\
\hline \multirow[t]{2}{*}{\begin{tabular}{|l} 
Lopez-Olivo et al. \\
(2011) [18]
\end{tabular}} & \multirow[t]{2}{*}{ OA } & \multirow[t]{2}{*}{ Primary TKA } & \multirow[t]{2}{*}{$65(9)$} & \multirow[t]{2}{*}{65} & \multirow[t]{2}{*}{232} & \multirow[t]{2}{*}{6 months } & \multirow[t]{2}{*}{$\begin{array}{l}\text { WOMAC pain and function } \\
\text { at } 6 \text { months }\end{array}$} & \multirow[t]{2}{*}{\begin{tabular}{|l} 
Multiple \\
regression \\
modelling
\end{tabular}} & \multirow[t]{2}{*}{ NONE } & $\begin{array}{l}\text { WOMAC pain } \\
\text { More education (+): } \beta=-0.17, p=0.01 \\
\text { More comorbidities }(-)=0=0.17, \mathrm{p}=0.008 \\
\text { More problem solving-style coping }(+) ; \beta=-0.14, \mathrm{p} \\
=0.03 \\
\text { More dysfunctional coping }(-) \text { : } \\
\beta=0.13, \mathrm{p}=0.04 \\
\text { More internal belief of control over health }(+): \beta= \\
-0.14, \mathrm{p}=0.02\end{array}$ & $\mid \begin{array}{l}\text { WOMAC pain } \\
\text { BMII } \\
\text { Baseline pain level }\end{array}$ \\
\hline & & & & & & & & & & $\begin{array}{l}\text { WOMAC function } \\
\text { More frequent availability of tangible support (+): } \\
\beta=-0.15, p=0.01 \\
\text { Worse depressive state }(-) ; \beta=0.15, p=0.02 \\
\text { More problem solving-style coping }(+): \beta=-0.20, p \\
=0.001 \\
\text { Lower baseline function level }(-) \text { : } \beta=0.25, p \\
=0.0001\end{array}$ & $\begin{array}{l}\text { WOMAC function } \\
\text { BMI } \\
\text { Comorbidities } \\
\end{array}$ \\
\hline
\end{tabular}




\begin{tabular}{|c|c|c|c|c|c|c|c|c|c|c|c|}
\hline \multirow[t]{2}{*}{ Study } & \multicolumn{4}{|c|}{ Participants } & \multirow{2}{*}{$\begin{array}{c}\text { Number } \\
\text { of } \\
\text { patients }\end{array}$} & \multirow{2}{*}{$\begin{array}{c}\text { Follow-up } \\
\text { period }\end{array}$} & \multirow[b]{2}{*}{ Outcome Measure } & \multirow{2}{*}{$\begin{array}{c}\text { Statistical } \\
\text { method }\end{array}$} & \multirow[b]{2}{*}{ Statistical adjustment } & \multicolumn{2}{|l|}{ Results } \\
\hline & Diagnosis & $\begin{array}{l}\text { Type of } \\
\text { Surgery }\end{array}$ & \begin{tabular}{|c|}
$\begin{array}{c}\text { Mean Age } \\
\text { (SD) }\end{array}$ \\
\end{tabular} & \begin{tabular}{|c|} 
Gender \\
(\%female $)$
\end{tabular} & & & & & & Significant Determinants & Non-significant Determinants \\
\hline \multirow[t]{3}{*}{\begin{tabular}{|l} 
Neuburger et al. \\
(2013) [23]
\end{tabular}} & \multirow[t]{3}{*}{$\mathrm{OA}(90 \%)$} & \multirow[t]{3}{*}{$\begin{array}{l}\text { Primary TKA } \\
(95 \%) \\
\text { Revision TKA } \\
(5 \%)\end{array}$} & \multirow[t]{3}{*}{\begin{tabular}{|l|}
$N / A$ \\
\end{tabular}} & \multirow[t]{3}{*}{$57 \%$} & \multirow[t]{3}{*}{62,303} & \multirow[t]{3}{*}{6 months } & \multirow[t]{3}{*}{ Total OKS score at 6 months } & \multirow[t]{3}{*}{$\begin{array}{l}\text { Logistic } \\
\text { regression } \\
\text { analysis }\end{array}$} & \begin{tabular}{|l} 
Model 1: \\
Age \\
Sex \\
Ethinicity \\
Self-reported comorbid \\
conditions \\
Self-reported general \\
health \\
Primary OA \\
Primary TKA or \\
revision TKA \\
Hospital \\
\end{tabular} & $\begin{array}{l}\text { Model 1: } \\
\text { More social deprivation (2nd quintile vs. 1st } \\
\text { quintite) (-): } \beta=-0.7,95 \%-0.9 \text { to }-0.5 \\
\text { More social deprivation (3rd quintile vs. 1st } \\
\text { quintile }(-) ;=-1.1,95 \%-1.3 \text { to }-0.9 \\
\text { More social deprivation (4th quintile vs. 1st } \\
\text { quintile) }(-) ; \beta=-2.2,95 \%-2.4 \text { to }-2.0 \\
\text { More social deprivation (thth quintile vs. 1st } \\
\text { quintile) (-): } \beta=-3.5,95 \%-3.8 \text { to }-3.3\end{array}$ & \begin{tabular}{|l} 
Model 1: \\
NONE
\end{tabular} \\
\hline & & & & & & & & & \begin{tabular}{|l|} 
Model 2: \\
Age \\
Sex \\
Ethnicity \\
Self-reported comorbid \\
conditions \\
Self-reported general \\
health \\
Primary OA \\
Primary TKA or \\
revision TKA \\
Hospital \\
Preoperative OKS \\
Longstanding problems \\
\end{tabular} & $\begin{array}{l}\text { Model 2: } \\
\text { More social deprivation (2nd quintile vs. 1st } \\
\text { quintilie) (-): } \beta=-0.4,95 \%-0.6 \text { to }-0.2 \\
\text { More social deprivation (3rd quintile vs. 1st } \\
\text { quintile) (-): } \beta=-0.6,95 \%-0.8 \text { to }-0.4 \text {. } \\
\text { More social deprivation (4th quintile vs. 1st } \\
\text { quintile) (-): } \beta=-1.5,95 \%-1.8 \text { to }-1.3 \\
\text { More social deprivation (5th quintile vs. } 1 \text { st } \\
\text { quintile) (-): } \beta=-2.4,95 \%-2.7 \text { to }-2.2 \\
\end{array}$ & \begin{tabular}{|l} 
Model 2: \\
NONE
\end{tabular} \\
\hline & & & & & & & & & \begin{tabular}{|l|} 
Model 3: \\
NONE
\end{tabular} & 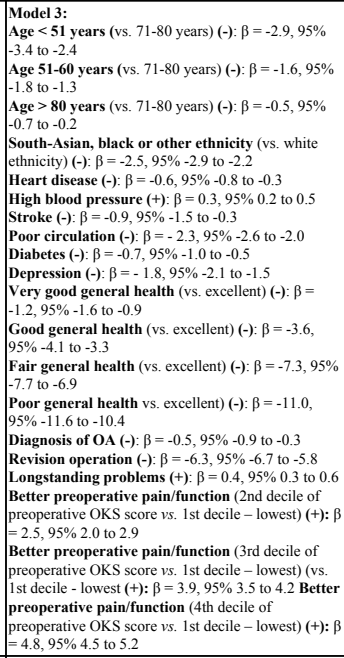 & \begin{tabular}{|l}
$\begin{array}{l}\text { Model 3: } \\
\text { Gender Age 61-70 years (vs. } \\
\text { 71-80 years) } \\
\text { Lung disease } \\
\text { Cancer }\end{array}$ \\
\\
\\
\end{tabular} \\
\hline \begin{tabular}{|l} 
Papakostidou et \\
al. (2012) [28]
\end{tabular} & $\mathrm{OA}(96 \%)$ & Primary TKA & $\begin{array}{l}69.17 \\
(6.69)\end{array}$ & 79.4 & 204 & 12 months & $\begin{array}{l}\text { WOMAC pain and function } \\
\text { at } 12 \text { months }\end{array}$ & $\begin{array}{l}\text { General linear } \\
\text { modelling }\end{array}$ & NONE & $\begin{array}{l}\text { WOMAC pain } \\
\text { Higher pre-intervention pain (-): Diff }=0.10,95 \% \\
\text { C1 } 0.02 \text { to } 2.29\end{array}$ & $\begin{array}{l}\text { WOMAC pain } \\
\text { Gender Age BMI Education } \\
\text { Social support Residence }\end{array}$ \\
\hline & & & & & & & & & & $\begin{array}{l}\text { WOMAC function } \\
\text { Lower pre-intervention function (-): Diff }=0.17 \text {, } \\
95 \% \mathrm{Cl} 0.06 \text { to } 0.28\end{array}$ & \begin{tabular}{|l|} 
WOMAC function \\
Gender Age BMI Education \\
Social support Residence
\end{tabular} \\
\hline $\begin{array}{l}\text { Perruccio et al. } \\
\text { (2012) [19] }\end{array}$ & OA & $\begin{array}{l}\text { Primary } \\
\text { unilateral TKA }\end{array}$ & 65 & 65 & 494 & 12 months & $\begin{array}{l}\text { WOMAC pain and function } \\
\text { at } 12 \text { months }\end{array}$ & \begin{tabular}{|l} 
Multiple linear \\
regression
\end{tabular} & NONE & $\begin{array}{l}\text { Pain } \\
\text { Symptomatic ankles/feet/toes (-): } \beta=1.24,95 \% \mathrm{CI} \\
0.04 \text { to } 2.00 \\
\text { Symptomatic neck (-): } \beta=1.07,95 \% \mathrm{CI} 0.17 \text { to } \\
1.98 \\
\text { Higher pre-surgery knee pain (-): } \beta=0.34,95 \% \mathrm{CI} \\
0.24 \text { to } 0.45 \\
\end{array}$ & \begin{tabular}{|l|} 
Pain \\
Age \\
Gender \\
Education \\
BMI \\
Comorbidity count \\
Symptomatic contralateral knee \\
Symptomatic hips \\
Symptomatic \\
elbows wristshands \\
Symptomatic shoulder \\
Symptomatic spine/lower back \\
\end{tabular} \\
\hline & & & & & & & & & & $\begin{array}{l}\text { Physical function } \\
\text { Symptomatic ankles/feet/toes (-): } \beta=3.14,95 \% \mathrm{CI} \\
0.69 \text { to } 5.59 \\
\text { Symptomatic neck (-): } \beta=3.46,95 \% \mathrm{CI} 0.54 \text { to } \\
6.38 \\
\text { Higher Pre-surgery knee function (+): } \beta=0.41 \text {, } \\
95 \% \text { CI } 0.31 \text { to } 0.50 \\
\\
\end{array}$ & \begin{tabular}{|l|} 
Physical function \\
Age \\
Gender \\
Education \\
Overweight \\
BMI \\
Comorbidity count \\
Symptomatic contralateral knee \\
Symptomatic hips \\
Symptomatic \\
elbows wristshands \\
Symptomatic shoulder \\
Symptomatic spine/lower back \\
\end{tabular} \\
\hline \begin{tabular}{|l} 
Rajgopal et al. \\
(2008) [20]
\end{tabular} & $\mathrm{OA}$ & $\begin{array}{l}\text { Primary TKA } \\
\text { (7.1\% with } \\
\text { history of } \\
\text { contralateral } \\
\text { TKA) }\end{array}$ & $\mathrm{N} / \mathrm{A}$ & 59.3 & 550 & 1 year & $\begin{array}{l}\text { Total WOMAC score at } 1 \\
\text { year }\end{array}$ & $\begin{array}{l}\text { Multiple linear } \\
\text { regression }\end{array}$ & NONE & $\begin{array}{l}\text { Higher baseline mental health }(+): \beta=0.210,95 \% \\
\text { CI } 0.063 \text { to } 357 \\
\text { Charrley Class C (-): } \beta=-4.897,95 \% \mathrm{CI}-8.701 \text { to } \\
-1.093 \\
\text { Higher baseline wOMAC score (+): } \beta=0.301 \text {, } \\
95 \% \mathrm{CI} 0.202 \text { to } 0.399 \\
\text { BMI } \geq 40 \text { (-): } \beta=-5.188,95 \% \mathrm{Cl}-9.771 \text { to }-0.606\end{array}$ & \begin{tabular}{|l|} 
Age \\
Gender \\
Prior contralateral TKA \\
BMI \\
\end{tabular} \\
\hline $\begin{array}{l}\text { Ramaesh et al. } \\
\text { (2013) [29] }\end{array}$ & Arthrosis & TKA & 70.5 & 58 & 205 & 1 year & Oxford Knee Score at 1 year & $\begin{array}{l}\text { Multiple linear } \\
\text { regression }\end{array}$ & NONE & $\begin{array}{l}\text { More comorbidity (-): } \mathrm{B}=-1.77,95 \% \mathrm{CI}-2.35 \text { to } \\
-1.19 \\
\text { Better preoperative function/pain level (+): } \mathrm{B}= \\
0.26,95 \% \mathrm{CI} 0.10 \text { to } 0.43\end{array}$ & \begin{tabular}{|l} 
Age \\
Gender \\
Personality type
\end{tabular} \\
\hline \multirow{6}{*}{$\begin{array}{l}\text { Riddle et al. } \\
(2010)[22]\end{array}$} & \multirow[t]{6}{*}{$\mathrm{OA}$} & \multirow[t]{6}{*}{ Primary TKA } & \multirow[t]{6}{*}{63.7} & \multirow[t]{6}{*}{70.7} & \multirow[t]{6}{*}{157} & \multirow[t]{6}{*}{6 months } & \multirow{6}{*}{$\begin{array}{l}\text { WOMAC pain and function } \\
\text { change scores at } 6 \text { months }\end{array}$} & \multirow{6}{*}{$\begin{array}{l}\begin{array}{l}\text { Logistic } \\
\text { regression }\end{array} \\
\end{array}$} & \multirow{6}{*}{\begin{tabular}{|l|} 
Age \\
Gender \\
BMI \\
Comorbidity \\
Rheumatoid arthritis \\
status \\
Race/ethnicity \\
Preoperative WOMAC \\
pain score
\end{tabular}} & WOMAC pain score & WOMAC pain score \\
\hline & & & & & & & & & & \begin{tabular}{|l|} 
Model 1: change by $<50 \%$ \\
Greater pain catastrophizing (PCS score $\geq 16)(-)$ : \\
OR $=2.67,95 \%$ CI 1.2 to 6.1
\end{tabular} & \begin{tabular}{|l|l|} 
Model 1 \\
None
\end{tabular} \\
\hline & & & & & & & & & & \begin{tabular}{|l|} 
Model 2: change $\leq 4$ points \\
Greater pain catastrophizing (PCS score $\geq 16)(-):$ \\
OR $=6.04,95 \%$ CI 1.75 to 20.82
\end{tabular} & \begin{tabular}{|l|}
$\begin{array}{l}\text { Model 2 } \\
\text { Self-efficacy } \\
\text { Kinesiophobia }\end{array}$ \\
\end{tabular} \\
\hline & & & & & & & & & & \begin{tabular}{|l|} 
WOMAC function score \\
\end{tabular} & WOMAC function score \\
\hline & & & & & & & & & & \begin{tabular}{|l|} 
Model 1: change by $<50 \%$ \\
None
\end{tabular} & \begin{tabular}{|l|} 
Model 1: change by $<50 \%$ \\
None
\end{tabular} \\
\hline & & & & & & & & & & $\begin{array}{l}\text { Model 2: change } \leq 15 \text { points } \\
\text { None }\end{array}$ & $\begin{array}{l}\text { Model 2: change } \leq 15 \text { points } \\
\text { None }\end{array}$ \\
\hline
\end{tabular}




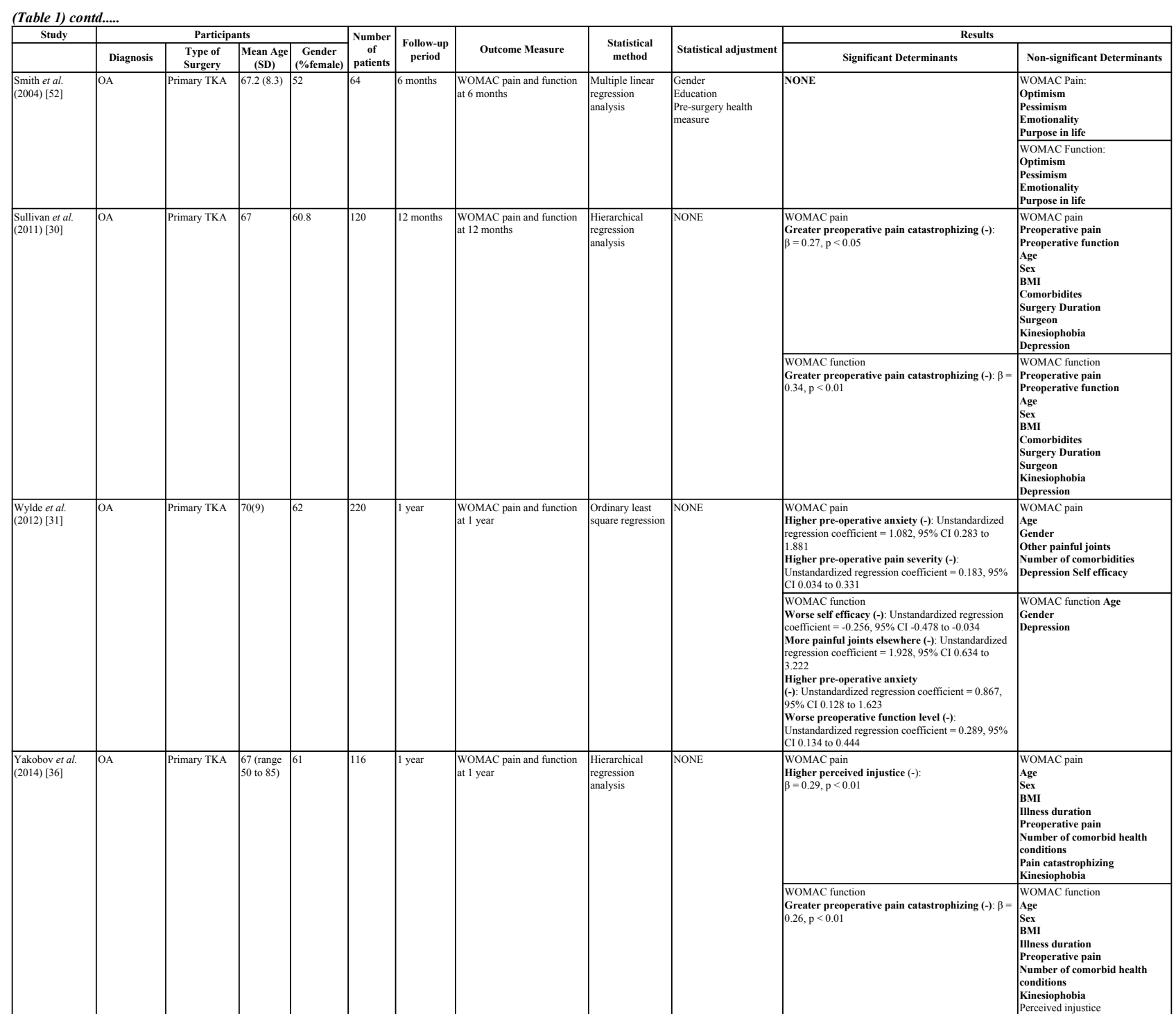

(+): determinant of successful outcome; (-): determinant of poor outcome; ASA -American Society of Anaestesiologists; ISTC - Independent Sector Treatment Centre; NHS - National Health Services; OR - Odds Ratio; QoL - quality of life; RA - Rheumatoid arthritis; UKA - unicompartmental knee arthroplasty; $\beta$ - regression coefficient.

The risk of bias and the methodological quality of the included studies was assessed using a modified version of the Methodology Checklist for Prognostic Studies developed by Hayden et al. (2003) [14]. This tool includes six items: "Study participation", 'Study attrition", "Prognostic factor measurement", 'Outcome measurement", "Confounding measurement and account" and "Analysis". Each item is evaluated according to its risk of potential bias: "yes" indicates a low risk of bias, "no" indicates a high risk of bias and "unclear" indicates an unclear or an unknown risk given the information available. For each item of the checklist, a score of 2 was given if a low risk of bias was present, a score of 1 if the risk was judged unclear and 0 if the risk was high. For the "Study participation" item, a score of 1 was attributed if the study was retrospective in nature and that no information was available regarding patients not included in the study because of incomplete data. For the "Study attrition" item, a score of 0 was given automatically if the follow-up proportion at the relevant time-point was inferior to $80 \%$. A score of 0 was given for the "Confounding measurement and account" item if confounding factors such as age, gender and body mass index (BMI) were not accounted for in the multivariate analysis. 


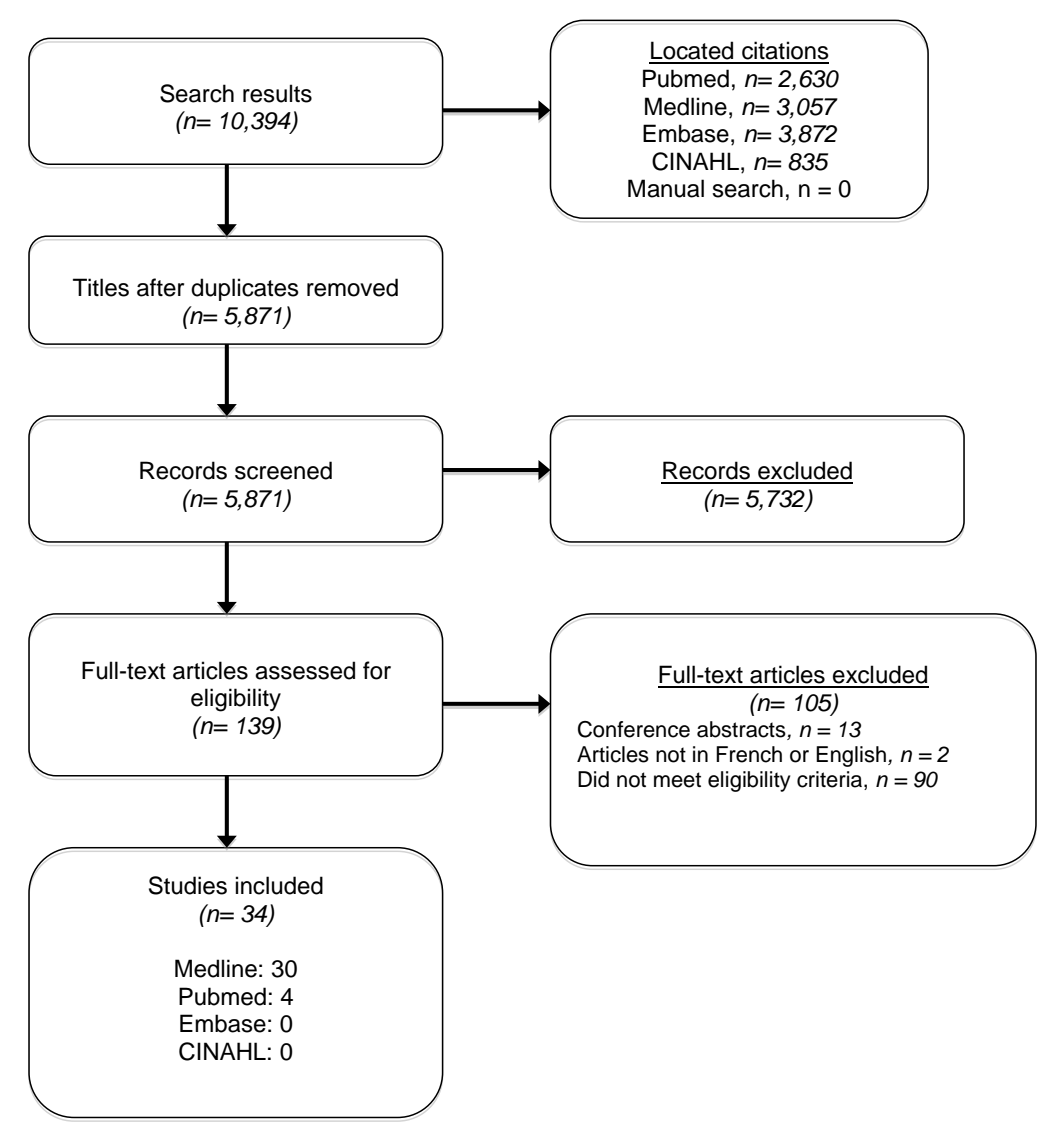

Fig. (1). Flowchart of the literature search.

\section{Data Synthesis}

Determinants of TKA outcomes were summarized based on whether results were reported as postoperative change or postoperative status, and whether pain and function were assessed as separate or combined constructs. Given the nature of the study designs and the heterogeneity of included studies in terms of depended and independent variables' constructs and definitions, as well as variations in follow-up periods, only a qualitative synthesis of results was performed.

\section{RESULTS}

\section{Description of the Included Studies}

Initial literature search yielded 139 full-text articles for assessment of eligibility. After further exclusion of 106 fulltext articles for reasons presented in Fig. (1), 33 manuscripts were included. Table 1 indicates relevant characteristics of the included studies. Results from two manuscripts are presented conjointly because of analyses performed on the same cohort $[9,15]$. The WOMAC was the validated tool used to measure postoperative pain and/or function in 24 studies, whereas the Oxford Knee Score (OKS) was employed in 9 studies. Nine studies have employed the change in pain and/or function after the surgery as an outcome measure. Postoperative raw scores at follow-up were considered as a measure of outcome in 25 studies. Six studies had a sample size smaller than 100 and 13 had a sample size greater than 500 patients. Only six studies presented a power calculation or considered a way of estimating required sample sizes [16 - 20].

\section{Methodological Quality of the Included Studies}

Table 2 indicates the methodological quality scores of the included studies after consensus. Mean total score for the methodological quality was $80.7 \%$ (SD $12.2 \%$ ). No study received lower than $58.3 \%$ and four studies were graded $100 \%[17,19,21,22]$. Overall, these results indicate a moderate-to-high methodological quality. 
Three domains of the methodology appraisal ("Prognostic factor measurement", "Outcome measurement" and "Analysis") scored on average the maximal possible grade. The domain with the worse mean score (1.00, SD 1.02) was "Confounding measurement and account", with 17 studies not accounting for age, gender or BMI or other potential confounding factor in the multivariate analyses. A noteworthy number of studies (11 out of 33) reported a follow-up proportion inferior to $80 \%$. This negatively impacted the study attrition domain.

Table 2. Methodological appraisal of the included studies.

\begin{tabular}{|c|c|c|c|c|c|c|c|}
\hline Included studies $(\mathrm{n}=33)$ & $\begin{array}{c}\text { Study } \\
\text { participation }\end{array}$ & $\begin{array}{c}\text { Study } \\
\text { attrition }\end{array}$ & $\begin{array}{c}\text { Prognostic factor } \\
\text { measurement }\end{array}$ & $\begin{array}{c}\text { Outcome } \\
\text { measurement }\end{array}$ & \begin{tabular}{|c|} 
Confounding \\
measurement and \\
account
\end{tabular} & Analysis & $\begin{array}{c}\text { Total } \\
\text { score } \\
/ 12 \\
\end{array}$ \\
\hline Alzharani et al. (2011) [33] & 2 & 1 & 2 & 2 & 2 & 2 & 11 \\
\hline Ayers et al. (2005) [42] & 2 & 1 & 2 & 2 & 0 & 2 & 9 \\
\hline Baker et al. (2012) [16] & 1 & 0 & 2 & 2 & 0 & 2 & 7 \\
\hline Caracciolo et al. (2005) [37] & 2 & 1 & 2 & 2 & 0 & 2 & 9 \\
\hline Clement et al. (2013) [35] & 1 & 1 & 2 & 2 & 0 & 2 & 8 \\
\hline Clement et al. (2013) [32] & 2 & 2 & 2 & 2 & 0 & 2 & 10 \\
\hline Clement et al. (2013) [40] & 1 & 1 & 2 & 2 & 0 & 2 & 8 \\
\hline Davis et al. (2008) [34] & 2 & 2 & 2 & 2 & 0 & 2 & 10 \\
\hline Desmeules et al. (2013) [17] & 2 & 2 & 2 & 2 & 2 & 2 & 12 \\
\hline Engel et al. (2004) [51] & 1 & 2 & 2 & 2 & 0 & 2 & 9 \\
\hline Escobar et al. (2007) [24] & 2 & 0 & 2 & 2 & 2 & 2 & 10 \\
\hline Fortin et al. (1999)9 \& (2002) [15] & 2 & 0 & 2 & 2 & 0 & 2 & 8 \\
\hline Gandhi et al. (2010) [39] & 2 & 1 & 2 & 2 & 2 & 2 & 10 \\
\hline Gandhi et al. (2013) [21] & 2 & 2 & 2 & 2 & 2 & 2 & 12 \\
\hline Hanusch et al. (2013) [27] & 2 & 2 & 2 & 2 & 0 & 2 & 10 \\
\hline Jones et al. (2001) [41] & 2 & 1 & 2 & 2 & 2 & 2 & 11 \\
\hline Jones et al. (2003) [10] & 2 & 0 & 2 & 2 & 2 & 2 & 10 \\
\hline Judge et al. (2012) [25] & 2 & 0 & 2 & 2 & 2 & 2 & 10 \\
\hline Kauppila et al. (2011) [4] & 2 & 2 & 2 & 2 & 0 & 2 & 10 \\
\hline Lingard et al. (2004) [26] & 2 & 0 & 2 & 2 & 2 & 2 & 10 \\
\hline Lingard et al. (2007) [38] & 2 & 0 & 2 & 2 & 0 & 2 & 8 \\
\hline Lopez-Olivo et al. (2011) [18] & 2 & 1 & 2 & 2 & 2 & 2 & 11 \\
\hline Neuburger et al. (2013) [23] & 1 & 0 & 2 & 2 & 0 & 2 & 7 \\
\hline Papakostidou et al. (2012) [28] & 2 & 1 & 2 & 2 & 2 & 2 & 11 \\
\hline Perruccio et al. (2012) [19] & 2 & 2 & 2 & 2 & 2 & 2 & 12 \\
\hline Rajgopal et al. (2008) [20] & 2 & 1 & 2 & 2 & 2 & 2 & 11 \\
\hline Ramaesh et al. (2013) [29] & 1 & 2 & 2 & 2 & 0 & 2 & 9 \\
\hline Riddle et al. (2010) [22] & 2 & 2 & 2 & 2 & 2 & 2 & 12 \\
\hline Smith et al. (2004) [52] & 1 & 0 & 2 & 2 & 0 & 2 & 7 \\
\hline Sullivan et al. (2011) [30] & 2 & 0 & 2 & 2 & 2 & 2 & 10 \\
\hline Wylde et al. (2012) [31] & 1 & 2 & 2 & 2 & 0 & 2 & 9 \\
\hline Yakobov et al. (2014) [36] & 1 & 0 & 2 & 2 & 2 & 2 & 9 \\
\hline TOTAL $($ mean \pm SD) $/ 12$ & $1.71 \pm 0.46$ & $1.00 \pm 0.84$ & $2.00 \pm 0.0$ & $2.00 \pm 0.0$ & $1.00 \pm 1.02$ & $2.00 \pm 0.0$ & $\begin{array}{c}9.69 \pm \\
1.47 \\
\end{array}$ \\
\hline TOTAL $($ mean \pm SD) $/ 100$ & $85.4 \pm 23.1$ & $50.0 \pm 42.1$ & $100 \pm 0.0$ & $100 \pm 0.0$ & $50.0 \pm 50.8$ & $100 \pm 0.0$ & $\begin{array}{c}80.7 \pm \\
12.2\end{array}$ \\
\hline
\end{tabular}

Study participation: The study sample represents the population of interest with regard to key characteristics, sufficient to limit potential bias to the results

Study attrition: Loss to follow-up is unrelated to key characteristics (that is, the study data adequately represent the sample), sufficient to limit potential bias

Prognostic factor measurement: The prognostic factor of interest is adequately measured in study participants, sufficient to limit potential bias Outcome measurement: The outcome of interest is adequately measured in study participants, sufficient to limit bias Confounding measurement and account: Important potential confounders are appropriately accounted for, limiting potential bias with respect to the prognostic factor of interest Analysis: The statistical analysis is appropriate for the design of the study, limiting potential for the presentation of invalid results

SD: standard deviation 


\section{Preoperative Determinants of TKA Pain and Function Outcomes}

\section{Demographic Determinants}

Fifteen studies investigated the association of age at the time of surgery and postoperative status. Neuburger et al. (2012) mention that being less than 60 years old is a significant determinant of poorer total OKS score at 6 months [23]. However, the same study reports that being older than 80 years old was also related to worse total OKS score at 6 months. Four more studies identified older age at the time of surgery as a factor associated with worse functional level following TKA [10, 24 - 26]. Nevertheless, ten studies report no significant effect of age on postoperative pain and function status $[9,15,19,20,24,27$ - 31]. Eleven studies did not report a significant relationship between gender and TKA outcomes $[9,10,15,19,23,24,27$ - 29, 32, 33]. The two that found a significant association seem to yield more consistent results regarding the deleterious effect of female gender on TKA pain and function outcomes [25, 26].

Only limited evidence can be extracted regarding demographic determinants of postoperative change in terms of pain or function. In regards to gender, one study identified male gender to be associated with a smaller change in the $12-$ month WOMAC function score [4]. Alzharani et al. (2011) report that male patients were 0.72 times more likely to not achieve the minimal clinically important difference (MCID) for total OKS score 1 year after TKA compared to women, i.e. female gender is a determinant of unsatisfactory outcome [33]. Baker et al. (2012) suggest that younger age is associated with less improvement on the total OKS score recorded 6 to 12 months postoperatively, whereas Alzharani et al. (2011) indicate that older age is associated with lower odds of attaining the MCID of the total WOMAC score 1 year following TKA $[16,33]$.

\section{Socioeconomic Determinants}

Although scarce, the evidence regarding socioeconomic factors seems to point to several significant findings only in the case of the outcomes measured as postoperative status. Greater social deprivation was identified in two studies as a determinant of worse pain and functional limitation when simultaneously controlling for multiple confounding factors $[23,25]$. A lower income was linked to a worse WOMAC pain score at 12 months post-operatively [34]. A lower educational status has been associated with better pain levels at 6 months in a study by Lopez-Olivo et al. (2012). However, six studies report no significant effect of education on either pain or function following TKA $[9,15,17,18$, $28,34]$.

\section{Psychosocial Determinants}

Several studies that were included in the review were dedicated at exploring the relationship between possible psychosocial determinants and TKA outcomes measured as postoperative status. Presence or higher levels of anxiety and/or depression have been consistently identified as significant determinants of worse TKA outcomes in six of the included studies [23, 25, 27, 31, 32, 35]. Two studies report that greater preoperative pain catastrophizing is linked to worse pain and disability 12 months after TKA [30,36]. Escobar et al. (2007) identified absence of social support to be related to worse 6-month pain and function levels [24]. Other significant psychosocial variables associated to pain and function status following TKA are presented in Table $\mathbf{3}$.

In terms of postoperative change, Riddle et al. (2010) determined that greater pain catastrophizing was related to higher odds of not achieving an improvement of 50\% in the pain domain of the WOMAC at 6 months as well as not attaining a change greater than 4 points out of 20 on the WOMAC pain score at 6 months [22]. A previous diagnosis of depression and higher levels of depression/anxiety as measured by the EuroQ5D questionnaire were related to a smaller change on the 6 to 12 month total OKS score [16].

Table 3. Significant pre-operative determinants of poor outcomes as measured by pain and/or function status at 6 weeks to 2 years following TKA.

\begin{tabular}{|c|c|c|c|c|c|c|}
\hline $\begin{array}{c}\text { Determinant } \\
\text { type }\end{array}$ & PAIN & Studies & FUNCTION & Studies & $\begin{array}{c}\text { PAIN \& FUNCTION } \\
\text { combined }\end{array}$ & Studies \\
\hline \multirow{4}{*}{ Demographic } & Younger age & [24] & Younger age & [10] & Female gender & {$[23,25]$} \\
\hline & \multirow[t]{3}{*}{ Female gender } & \multirow[t]{3}{*}{ [26] } & Older age & {$[25,26]$} & $\begin{array}{l}\text { South-Asian, black or } \\
\text { other non-white ethnicity }\end{array}$ & {$[23]$} \\
\hline & & & Female gender & {$[25]$} & Younger age & {$[23]$} \\
\hline & & & Single, separated or divorced & [17] & Older age & {$[23]$} \\
\hline
\end{tabular}




\begin{tabular}{|c|c|c|c|c|c|c|}
\hline $\begin{array}{c}\text { Determinant } \\
\text { type }\end{array}$ & PAIN & Studies & FUNCTION & Studies & $\begin{array}{c}\text { PAIN \& FUNCTION } \\
\text { combined }\end{array}$ & Studies \\
\hline \multirow[t]{3}{*}{ Socioeconomic } & Low income & {$[34]$} & Unemployed or retired & {$[17]$} & \multirow{3}{*}{$\begin{array}{l}\text { Greater social } \\
\text { deprivation }\end{array}$} & \multirow[t]{3}{*}[23,25]{} \\
\hline & Greater social deprivation & {$[25]$} & \multirow[t]{2}{*}{ Greater social deprivation } & \multirow[t]{2}{*}[25]{} & & \\
\hline & Lower education level & {$[18]$} & & & & \\
\hline \multirow[t]{13}{*}{ Psychosocial } & Lower coping efficacy & {$[51]$} & Lower coping efficacy & {$[51]$} & Presence of depression & $\begin{array}{c}{[23,25,32,} \\
35]\end{array}$ \\
\hline & High arthritis helplessness & {$[51]$} & Absence of social support & {$[24]$} & \multirow[t]{12}{*}{ Higher anxiety level } & \multirow[t]{12}{*}[25,27]{} \\
\hline & Higher pessimism & {$[51]$} & Higher anxiety & {$[25,31]$} & & \\
\hline & $\begin{array}{l}\text { Lower expected chance of } \\
\text { recovery }\end{array}$ & {$[51]$} & Higher depression level & {$[18,25]$} & & \\
\hline & $\begin{array}{l}\text { Lower expected change in quality } \\
\text { of life }\end{array}$ & {$[51]$} & $\begin{array}{l}\text { Less frequent availability of } \\
\text { tangible support }\end{array}$ & {$[18]$} & & \\
\hline & Absence of social support & {$[24]$} & $\begin{array}{l}\text { Less problem-solving coping } \\
\text { style }\end{array}$ & {$[18]$} & & \\
\hline & Higher anxiety level & {$[25,31]$} & Greater pain catastrophizing & {$[30,36]$} & & \\
\hline & Higher depression level & {$[25]$} & \multirow[t]{6}{*}{ Worse self-efficacy } & \multirow[t]{6}{*}{ [32] } & & \\
\hline & $\begin{array}{l}\text { Less problem-solving coping } \\
\text { style }\end{array}$ & {$[18]$} & & & & \\
\hline & More dysfunctional coping & {$[18]$} & & & & \\
\hline & $\begin{array}{l}\text { Less internal belief of control } \\
\text { over health }\end{array}$ & {$[18]$} & & & & \\
\hline & Greater pain catastrophizing & {$[30]$} & & & & \\
\hline & Greater perceived injustice & {$[37]$} & & & & \\
\hline \multirow[t]{15}{*}{ Clinical } & Worse pain level & $\begin{array}{c}{[9,15,17} \\
29,24-26 \\
28,32]\end{array}$ & Worse function level & $\begin{array}{c}{[9,10,15,} \\
17-19,24- \\
26,28,31, \\
37]\end{array}$ & Presence of back pain & {$[33,35]$} \\
\hline & Presence of back pain & {$[24,26]$} & Presence of back pain & {$[24]$} & $\begin{array}{l}\text { Worse pain/function } \\
\text { levels }\end{array}$ & $\begin{array}{l}{[20,23,25,} \\
27,29,35]\end{array}$ \\
\hline & Greater comorbidity & $\begin{array}{c}{[18,24,26,} \\
38]\end{array}$ & Greater comorbidity & {$[10,24,26]$} & Worse mental health & {$[20,33,35]$} \\
\hline & Worse mental health & {$[25]$} & Worse mental health & {$[10,26,38]$} & $\begin{array}{l}\text { Worse general health } \\
\text { status }\end{array}$ & {$[23]$} \\
\hline & OA diagnosis & [19] & Use of walking devices & {$[10]$} & Vascular comorbidity & {$[23,26]$} \\
\hline & Symptomatic ankles/feet/toes & [19] & Higher BMI & {$[25,26]$} & Obesity & {$[39]$} \\
\hline & \multirow[t]{9}{*}{ Symptomatic neck } & \multirow[t]{9}{*}{ [19] } & Symptomatic ankles/feet/toes & {$[19]$} & Higher BMI & {$[20,25]$} \\
\hline & & & Symptomatic neck & [19] & OA diagnosis & {$[23,25]$} \\
\hline & & & More painful joints elsewhere & {$[31]$} & Greater comorbidity & {$[20,29]$} \\
\hline & & & \multirow[t]{6}{*}{ Restricted knee flexion } & \multirow[t]{6}{*}[26]{} & Heart disease & {$[23]$} \\
\hline & & & & & $\begin{array}{l}\text { Absence of high blood } \\
\text { pressure }\end{array}$ & {$[23]$} \\
\hline & & & & & Stroke & {$[23]$} \\
\hline & & & & & Diabetes & {$[23]$} \\
\hline & & & & & Revision surgery & {$[23]$} \\
\hline & & & & & $\begin{array}{l}\text { Shorter duration of } \\
\text { symptoms }\end{array}$ & {$[23]$} \\
\hline Surgical & Cruciate-retaining implant & {$[17]$} & NONE & NONE & NONE & NONE \\
\hline
\end{tabular}

\section{Clinical Determinants}

The investigation of the association between clinical characteristics and TKA outcomes measured as postoperative status has received a great deal of attention. One of the most studied potential determinants of knee pain and function following TKA is the baseline, preoperative levels of the respective variables. Seventeen studies linked a poor preoperative status to a worse postoperative status in terms of pain and function $[9,10,15,17-20,23-25,27,28,31$, $32,35,37,38]$.

Poor preoperative mental health, as measured by the SF-36 questionnaire, has been associated to worse outcomes in seven studies [18, 20, 24, 26, 32, 35, 38]. Even if TKA is performed predominantly for patients with primary OA, two studies seem to point to a diagnosis of primary gonarthrosis as a determinant of worse outcomes, when compared to 
rheumatoid arthritis or other diagnoses [23, 25]. Higher baseline BMI has been linked to poorer functional results as well as to worse outcomes of pain and function combined in four studies [20, 25, 26, 39]. Five studies identified the presence of back pain before surgery to be related to substandard pain and function status after TKA [19, 24, 28, 32, 35]. Regardless of whether comorbidity was measured as the influence of individual comorbidities, of the number of comorbidities per patient or when considering their severity and impact on patients' life, seven studies suggests it to be a significant determinant of worse outcomes in terms of pain and function following TKA [10, 20, 23, 24, 26, 29, 35]. More symptomatic joints, including ankle, feet, toes and neck were associated with greater level of pain and worse function 12 months after TKA in two studies [19, 31]. Other significant clinical determinants of TKA outcomes measured as postoperative status can be found in Table 3 .

Regarding outcomes measured as postoperative change, four studies report that better baseline levels of function and/or pain are related to lower levels of improvement following TKA. Jones et al. (2001) reported that lower preoperative pain was associated with smaller changes in functional abilities 6 months after the surgery [10]. A better preoperative total OKS score was related to a smaller change 6 to 12 months following TKA in two studies [16, 40]. Better preoperative function level was found to be a significant determinant of lower gains in functional abilities [4].

Greater comorbidity was shown to significantly determine lower changes in pain and functional status [16, 40, 41]. In particular, Kauppila et al. (2011) showed that presence of osteoporosis was associated with a smaller level of change in function and with decreased odds of attaining the OMERACT-OARSI set of responder criteria 12 months after surgery [4]. A study by Gandhi et al. (2013) revealed that a greater level of synovial fluid levels of three inflammatory markers (TNF- $\alpha$, MMP-13 and IL-6) were related to poor gains in physical function 2 years after TKA as measured by the WOMAC function score [21]. Other miscellaneous clinical determinants identified in the included studies were worse general health status (as measured by the American Society of Anaesthesiology grade), presence of self-reported disability and lower self-reported general health [16], greater preoperative bodily pain [41], worse mental health [40, 42] and presence of back pain [40].

\section{Surgical Determinants}

Only one of the included studies identified a significant surgical determinant of poor postoperative status as measured by pain levels at 6 months: cruciate-retaining implant [17]. Sullivan et al. (2011) studied the effect of surgery duration and of the identity of the surgeon on the 12-month WOMAC pain and function scales, but their analysis yielded non-significant results [30].

In terms of postoperative change, findings by Jones et al. (2001) indicate that cementless prosthesis is associated with a lower change in the WOMAC pain score 6 months after TKA [41]. A British study by Baker et al. (2012) evaluated the effect of different types of prosthesis brands on the improvement of the total OKS score 12 months following the intervention. They found that the NexGen prosthesis brand is related to greater improvements when compared to all the other brands used in their study (PFC, Genesis 2, AGC and Triathlon) [16]. The same study evaluated the effect of the type of hospital where the surgery was performed. They showed that surgeries performed at a National Health Services hospital are more likely to be associated with poor improvement than surgeries performed at an independent hospital or an Independent Sector Treatment Centre.

\section{DISCUSSION}

Because TKA clinical results are still suboptimal in a large percentage of patients, a better knowledge of determinants of pain and function following the intervention could help improve outcomes. The aim of our study was to systematically assess the literature reporting the determinants of pain and functional outcomes following primary unilateral TKA in patients with knee OA. Thirty-four studies with a moderate-to high mean methodological quality $(80.7 \%$, SD $12.2 \%)$ were included. Even if several significant determinants of pain and functional outcomes following TKA have been summarized by studies, no conclusions can be reached regarding the strength of the associations between significant determinants and TKA results because of heterogeneity of study methodologies and results.

\section{Strengths and Limitations of the Review}

The main strength of the present systematic review is the rigorousness of the inclusion criteria ensuring high quality of evidence of determinants compiled from four important databases. Moreover, focus on all types of determinants provides a comprehensive overview of all relevant variables with a significant relationship to TKA outcomes. 
The main limitation is the inability to pool the results into meta-analyses, resulting in the failure to conclude on the strength of association between patient factors and TKA outcomes due to the heterogeneity of the methodologies of the included studies. Moreover, the findings of the review do not necessarily apply to all patients undergoing TKA, namely those with a diagnosis different from OA, or undergoing bilateral or revision surgery. Also, the study does not review determinants of long-term outcomes. Finally, two studies had to be excluded because they were published in languages not mastered by the reviewers.

\section{Main Findings}

It is difficult to conclude to a significant association of any demographic determinant with TKA pain or functional outcomes based on the results of the included studies. Although female gender and older age were found significant in several studies, there is an overwhelming amount of evidence pointing to either an association in an opposite direction or to no relationship at all. These findings contrast the ones by Santaguida et al. (2008) in their systematic review. We therefore suggest that according to the available evidence, patients should not be denied surgery based on gender or age.

Regarding socioeconomic determinants, greater social deprivation achieved statistical significance in both studies evaluating its association with TKA outcomes among patients in the United Kingdom [23, 25]. Patients with greater social deprivation may experience worse TKA outcomes because of an inequality in the continuity of care following discharge compared to patients with less deprivation [23]. Caution should be warranted regarding the generalizability of these findings however, as they may not apply to other countries, although the impact of social deprivation in terms of pain and function on other musculoskeletal disorders is well established [43, 44].

Psychosocial determinants with considerable evidence include the presence or a greater level of depression and/or anxiety. The previous review by Vissers et al. (2012) did not find definite evidence that supports the significance of this association. However, all of the seven studies included in our review that conclude to such a relationship were published after the beforementioned systematic review. The causes behind the significant association are not well understood; depressed patients might be less likely to participate actively in the rehabilitation process, thus experiencing worse outcomes [18]. Greater preoperative pain catastrophizing was also significantly associated to pain and functional outcomes after TKA, a finding consistent with the review by Vissers et al. (2012). It has been suggested that pain catastrophizing is linked with neurophysiological processes related to modulation of pain, and that greater levels of catastrophizing promote sensitization to pain [30].

The greatest amount of evidence is available for clinical determinants, the frontrunner being the relationship between worse or better preoperative levels in the respective dimensions (depending on the outcome being measured as postoperative patient state or change) and pain or functional outcomes. Although studies consistently refer to this relationship as a well-known fact, to our knowledge, this is the first systematic review underlining this fact. Our findings suggest that in the case of measuring outcome as a change in status, a higher preoperative status is related to a lower chance of improvement. In the case of measuring outcome as health status postoperatively, lower preoperative status is related to worse outcome. The importance of these concepts relies in the dilemma encountered when employing this information clinically: should intervention be undertaken in patients with worse preoperative state in order to obtain greater gains or should TKA be performed in patients as early as possible before they deteriorate considerably in order to guarantee better status after the surgery? Unfortunately, there does not seem to be a consensus regarding this predicament, and our review only emphasizes its importance, as other authors have done as well [45].

Presence or greater levels of comorbidities were also related to a worse outcome after TKA. The reasons behind such a relationship are unclear. Patients with other comorbidities may not meet the demands of the intensive rehabilitation process following TKA, thus explaining their increased risk of poor surgical outcomes [26]. Several studies advocate that patients should receive appropriate counselling from their surgeon preoperatively according to the identity and number of their comorbidities [26,39]. Of interest, presence of back pain was associated to poor TKA outcomes as well. The mechanism behind this association is however uncertain. Back pain may impede postoperative recuperation and rehabilitation or it may directly affect how patients rate their condition in terms of knee pain and function on the WOMAC, or on other outcome measures [24].

Worse measures of general health were significantly related to poor TKA outcomes in a surprisingly consistent manner. Among the included studies, general health was mainly measured with the SF-36 questionnaire, and a poorer mental health domain in particular was consistently related to poor pain and function after TKA. This may underlie the importance of the overall health status, especially the extent of psychological distress, in selecting individuals for knee 
arthroplasty.

Limited evidence has been identified regarding surgical determinants of poor TKA outcomes. This may be due to the fact that surgical factors are traditionally investigated by studies employing a clinical trial methodology, whereas this review encompasses prognostic cohort studies. Association of surgical and technical factors with TKA outcomes is clearly a complex issue, and a different approach than the one employed by this review may be required to identify significant determinants.

No variable was consistently identified as non-significantly related to TKA outcome. The amount of evidence for certain significant determinants is nevertheless countered by numerous studies stating their non-significance and this inconsistency represents a limitation of the available literature. Several reasons behind this discrepancy can emerge. Firstly, a low sample size can impact the ability to detect a truly present statistically significant relationship; type II errors may effectively limit the findings. Also, the duration of follow-up may lead to a disagreement as a significant relationship may arise at a critical time-point following the surgery. Finally, the methodological quality of the studies can lead to heterogeneous results.

It has previously been suggested that the determinants of pain and function after TKA are not the same [25]. We attempted to appraise this by capturing the results of the included studies based on whether determinants were assessed for pain and function as separate dependent variables or part of a combined construct (Tables 3 and 4). On several occasions, individual studies that evaluated significant determinants of pain and function separately concluded that they indeed presented different determinants. However, when viewing the overall picture, the determinants of pain and function seem to be similar regardless of the method of measurement. This is most probably due to the overlap between the findings of the studies.

Table 4. Significant pre-operative determinants of poor outcomes as measured by change in pain and/or function status 6 weeks to 2 years following TKA.

\begin{tabular}{|c|c|c|c|c|c|c|}
\hline Determinant type & PAIN & Studies & FUNCTION & Studies & PAIN \& FUNCTION combined & Studies \\
\hline \multirow[t]{3}{*}{ Demographic } & \multirow[t]{3}{*}{ NONE } & \multirow[t]{3}{*}{ NONE } & \multirow[t]{3}{*}{ Male gender } & \multirow[t]{3}{*}[4]{} & Younger age & {$[16]$} \\
\hline & & & & & Older age & [33] \\
\hline & & & & & Female gender & [33] \\
\hline Socioeconomic & NONE & NONE & NONE & NONE & NONE & NONE \\
\hline \multirow[t]{2}{*}{ Psychosocial } & \multirow{2}{*}{$\begin{array}{l}\text { Greater pain } \\
\text { catastrophizing }\end{array}$} & \multirow[t]{2}{*}[22]{} & \multirow[t]{2}{*}{ NONE } & \multirow{2}{*}{ NONE } & Presence of depression & {$[16]$} \\
\hline & & & & & Greater depression level & {$[16]$} \\
\hline \multirow[t]{8}{*}{ Clinical } & $\begin{array}{l}\text { Greater levels of } \\
\text { inflammatory markers } \\
\text { - Greater synovial fluid } \\
\text { TNF- } \alpha \text { levels } \\
\text { - Greater synovial fluid } \\
\text { MMP-13 levels } \\
\text { - Greater synovial fluid } \\
\text { IL-6 levels }\end{array}$ & [21] & Better pain level & [41] & Better pain/function levels & {$[16,40]$} \\
\hline & \multirow[t]{7}{*}{ Higher bodily pain levels } & \multirow[t]{7}{*}{ [41] } & Greater comorbidity & [41] & Worse general health status & {$[16]$} \\
\hline & & & Higher bodily pain levels & [41] & Greater comorbidity & {$[16,40]$} \\
\hline & & & Worse mental health & {$[42]$} & Presence of self-reported disability & {$[16]$} \\
\hline & & & Presence of osteoporosis & {$[4]$} & Lower self-reported general health & [16] \\
\hline & & & \multirow[t]{3}{*}{ Better function level } & \multirow[t]{3}{*}{ [4] } & Presence of back pain & [41] \\
\hline & & & & & Worse mental health & {$[4]$} \\
\hline & & & & & Presence of osteoporosis & [4] \\
\hline \multirow[t]{4}{*}{ Surgical } & \multirow[t]{4}{*}{ Cementless prosthesis } & \multirow[t]{4}{*}{ [42] } & \multirow[t]{4}{*}{ NONE } & \multirow[t]{4}{*}{ NONE } & PFC prosthesis brand & [16] \\
\hline & & & & & Genesis 2 prosthesis brand & {$[16]$} \\
\hline & & & & & AGC prosthesis brand & [16] \\
\hline & & & & & Triathlon prosthesis brand & [16] \\
\hline Healthcare-related & NONE & NONE & NONE & NONE & $\begin{array}{l}\text { Surgery performed at a National Health Services } \\
\text { Hospital (United Kingdom) }\end{array}$ & [16] \\
\hline
\end{tabular}

As mentioned previously, outcomes after TKA are generally evaluated as a function of health change or of health state postoperatively [46 - 50]. In our review, we identified fewer studies evaluating determinants based on 
postoperative change. Generally, from the available evidence, determinants are similar between the two approaches, with the exception of the preoperative status as discussed previously.

\section{CONCLUSION}

Moderate-to-high methodological quality of included studies suggests that preoperative determinants of pain and function outcomes following TKA include greater social deprivation, the presence or a greater level of depression and/or anxiety, greater preoperative pain catastrophizing, preoperative pain or function levels, presence or greater levels of comorbidity, presence of back pain and lower general health. Consensus is however limited by contradictory results regarding the importance of several determinants. The heterogeneity in the measurement of the outcome limits the ability to generalize the magnitude of association of determinants with TKA outcomes. Further high-quality research and a more standardized reporting of results is required in order to elucidate with greater precision the identity of determinants of pain and function following TKA in order to provide the best possible care for patients with severe knee OA.

\section{CONFLICT OF INTEREST}

The authors confirm that this article content has no conflict of interest.

\section{ACKNOWLEDGEMENTS}

Declared none.

\section{REFERENCES}

[1] NIH Consensus Statement on total knee replacement. NIH Consens State Sci Statements 2003; 20(1): 1-34. [PMID: 17308549]

[2] Kurtz S, Ong K, Lau E, Mowat F, Halpern M. Projections of primary and revision hip and knee arthroplasty in the United States from 2005 to 2030. J Bone Joint Surg Am 2007; 89(4): 780-5.

[http://dx.doi.org/10.2106/JBJS.F.00222] [PMID: 17403800]

[3] Jones CA, Voaklander DC, Johnston DW, Suarez-Almazor ME. Health related quality of life outcomes after total hip and knee arthroplasties in a community based population. J Rheumatol 2000; 27(7): 1745-52. [PMID: 10914862]

[4] Kauppila A-M, Kyllönen E, Ohtonen P, Leppilahti J, Sintonen H, Arokoski JP. Outcomes of primary total knee arthroplasty: the impact of patient-relevant factors on self-reported function and quality of life. Disabil Rehabil 2011; 33(17-18): 1659-67. [http://dx.doi.org/10.3109/09638288.2010.543749] [PMID: 21184628]

[5] Liang MH, Cullen KE, Larson MG, et al. Cost-effectiveness of total joint arthroplasty in osteoarthritis. Arthritis Rheum 1986; $29(8)$ : 937-43. [http://dx.doi.org/10.1002/art.1780290801] [PMID: 3091041]

[6] Singh JA, O’Byrne M, Harmsen S, Lewallen D. Predictors of moderate-severe functional limitation after primary Total Knee Arthroplasty (TKA): 4701 TKAs at 2-years and 2935 TKAs at 5-years. Osteoarthritis Cartilage 2010; 18(4): 515-21. [http://dx.doi.org/10.1016/j.joca.2009.12.001] [PMID: 20060950]

[7] Wylde V, Hewlett S, Learmonth ID, Dieppe P. Persistent pain after joint replacement: prevalence, sensory qualities, and postoperative determinants. Pain 2011; 152(3): 566-72. [http://dx.doi.org/10.1016/j.pain.2010.11.023] [PMID: 21239114]

[8] Santaguida PL, Hawker GA, Hudak PL, et al. Patient characteristics affecting the prognosis of total hip and knee joint arthroplasty: a systematic review. Can J Surg 2008; 51(6): 428-36. [PMID: 19057730]

[9] Fortin PR, Penrod JR, Clarke AE, et al. Timing of total joint replacement affects clinical outcomes among patients with osteoarthritis of the hip or knee. Arthritis Rheum 2002; 46(12): 3327-30. [http://dx.doi.org/10.1002/art.10631] [PMID: 12483739]

[10] Jones CA, Voaklander DC, Suarez-Alma ME. Determinants of function after total knee arthroplasty. Phys Ther 2003; 83(8): 696-706. [PMID: 12882610]

[11] van Jonbergen H-P, Reuver JM, Mutsaerts EL, Poolman RW. Determinants of anterior knee pain following total knee replacement: a systematic review. Knee Surg Sports Traumatol Arthrosc 2014; 22(3): 478-99. [http://dx.doi.org/10.1007/s00167-012-2294-x] [PMID: 23160846]

[12] Vissers MM, Bussmann JB, Verhaar JA, Busschbach JJ, Bierma-Zeinstra SM, Reijman M. Psychological factors affecting the outcome of total hip and knee arthroplasty: a systematic review. Semin Arthritis Rheum 2012; 41(4): 576-88. [http://dx.doi.org/10.1016/j.semarthrit.2011.07.003] [PMID: 22035624]

[13] von Elm E, Altman DG, Egger M, Pocock SJ, Gøtzsche PC, Vandenbroucke JP. The Strengthening the Reporting of Observational Studies in 
Epidemiology (STROBE) statement: guidelines for reporting observational studies. Prev Med 2007; 45(4): $247-51$. [http://dx.doi.org/10.1016/j.ypmed.2007.08.012] [PMID: 17950122]

[14] Hayden JA, Côté P, Bombardier C. Evaluation of the quality of prognosis studies in systematic reviews. Ann Intern Med 2006; 144(6): 427-37. [http://dx.doi.org/10.7326/0003-4819-144-6-200603210-00010] [PMID: 16549855]

[15] Fortin PR, Clarke AE, Joseph L, et al. Outcomes of total hip and knee replacement: preoperative functional status predicts outcomes at six months after surgery. Arthritis Rheum 1999; 42(8): 1722-8. [http://dx.doi.org/10.1002/1529-0131(199908)42:8<1722::AID-ANR22>3.0.CO;2-R] [PMID: 10446873]

[16] Baker PN, Deehan DJ, Lees D, et al. The effect of surgical factors on early patient-reported outcome measures (PROMS) following total knee replacement. J Bone Joint Surg Br 2012; 94(8): 1058-66. [http://dx.doi.org/10.1302/0301-620X.94B8.28786] [PMID: 22844046]

[17] Desmeules F, Dionne CE, Belzile EL, Bourbonnais R, Champagne F, Frémont P. Determinants of pain, functional limitations and healthrelated quality of life six months after total knee arthroplasty: results from a prospective cohort study. BMC Sports Sci Med Rehabil 2013; 5: 2 . [http://dx.doi.org/10.1186/2052-1847-5-2] [PMID: 23566925]

[18] Lopez-Olivo MA, Landon GC, Siff SJ, et al. Psychosocial determinants of outcomes in knee replacement. Ann Rheum Dis 2011; 70(10): 1775-81. [http://dx.doi.org/10.1136/ard.2010.146423] [PMID: 21791452]

[19] Perruccio AV, Power JD, Evans HM, et al. Multiple joint involvement in total knee replacement for osteoarthritis: Effects on patient-reported outcomes. Arthritis Care Res (Hoboken) 2012; 64(6): 838-46. [http://dx.doi.org/10.1002/acr.21629] [PMID: 22570306]

[20] Rajgopal V, Bourne RB, Chesworth BM, MacDonald SJ, McCalden RW, Rorabeck CH. The impact of morbid obesity on patient outcomes after total knee arthroplasty. J Arthroplasty 2008; 23(6): 795-800. [http://dx.doi.org/10.1016/j.arth.2007.08.005] [PMID: 18534516]

[21] Gandhi R, Santone D, Takahashi M, Dessouki O, Mahomed NN. Inflammatory predictors of ongoing pain 2 years following knee replacement surgery. Knee 2013; 20(5): 316-8. [http://dx.doi.org/10.1016/j.knee.2012.10.015] [PMID: 23157967]

[22] Riddle DL, Wade JB, Jiranek WA, Kong X. Preoperative pain catastrophizing predicts pain outcome after knee arthroplasty. Clin Orthop Relat Res 2010; 468(3): 798-806. [http://dx.doi.org/10.1007/s11999-009-0963-y] [PMID: 19585177]

[23] Neuburger J, Hutchings A, Black N, van der Meulen JH. Socioeconomic differences in patient-reported outcomes after a hip or knee replacement in the English National Health Service. J Public Health (Oxf) 2013; 35(1): 115-24. [http://dx.doi.org/10.1093/pubmed/fds048] [PMID: 22729275]

[24] Escobar A, Quintana JM, Bilbao A, et al. Effect of patient characteristics on reported outcomes after total knee replacement. Rheumatology (Oxford) 2007; 46(1): 112-9. [http://dx.doi.org/10.1093/rheumatology/kel184] [PMID: 16735451]

[25] Judge A, Arden NK, Cooper C, et al. Predictors of outcomes of total knee replacement surgery. Rheumatology (Oxford) 2012; 51(10): 1804-13.

[http://dx.doi.org/10.1093/rheumatology/kes075] [PMID: 22532699]

[26] Lingard EA, Katz JN, Wright EA, Sledge CB, Kinemax Outcomes G. Predicting the outcome of total knee arthroplasty. J Bone Joint Surg Am 2004; 86-A(10): 2179-86. [PMID: 15466726]

[27] Hanusch BC, O’Connor DB, Ions P, Scott A, Gregg PJ. Effects of psychological distress and perceptions of illness on recovery from total knee replacement. Bone Joint J 2014; 96-B(2): 210-6. [http://dx.doi.org/10.1302/0301-620X.96B2.31136] [PMID: 24493186]

[28] Papakostidou I, Dailiana ZH, Papapolychroniou T, et al. Factors affecting the quality of life after total knee arthroplasties: a prospective study. BMC Musculoskelet Disord 2012; 13: 116. [http://dx.doi.org/10.1186/1471-2474-13-116] [PMID: 22748117]

[29] Ramaesh R, Jenkins P, Lane JV, Knight S, Macdonald D, Howie C. Personality, function and satisfaction in patients undergoing total hip or knee replacement. J Orthop Sci 2014; 19(2): 275-81. [http://dx.doi.org/10.1007/s00776-013-0509-8] [PMID: 24362559]

[30] Sullivan M, Tanzer M, Stanish W, et al. Psychological determinants of problematic outcomes following Total Knee Arthroplasty. Pain 2009; 143(1-2): 123-9. [http://dx.doi.org/10.1016/j.pain.2009.02.011] [PMID: 19304392]

[31] Wylde V, Dixon S, Blom AW. The role of preoperative self-efficacy in predicting outcome after total knee replacement. Musculoskelet Care 2012; 10(2): 110-8.

[http://dx.doi.org/10.1002/msc.1008] [PMID: 22368121] 
[32] Clement ND, MacDonald D, Simpson AH, Burnett R. Total knee replacement in patients with concomitant back pain results in a worse functional outcome and a lower rate of satisfaction. Bone Joint J 2013; 95-B(12): 1632-9. [http://dx.doi.org/10.1302/0301-620X.95B12.31684] [PMID: 24293592]

[33] Alzahrani K, Gandhi R, Debeer J, Petruccelli D, Mahomed N. Prevalence of clinically significant improvement following total knee replacement. J Rheumatol 2011; 38(4): 753-9. [http://dx.doi.org/10.3899/jrheum.100233] [PMID: 21239743]

[34] Davis ET, Lingard EA, Schemitsch EH, Waddell JP. Effects of socioeconomic status on patients' outcome after total knee arthroplasty. Int J Qual Health Care 2008; 20(1): 40-6. [http://dx.doi.org/10.1093/intqhe/mzm059] [PMID: 18174221]

[35] Clement ND, MacDonald D, Burnett R, Breusch SJ. Diabetes does not influence the early outcome of total knee replacement: a prospective study assessing the Oxford knee score, short form 12, and patient satisfaction. Knee 2013; 20(6): 437-41. [http://dx.doi.org/10.1016/j.knee.2013.07.009] [PMID: 23993274]

[36] Yakobov E, Scott W, Stanish W, Dunbar M, Richardson G, Sullivan M. The role of perceived injustice in the prediction of pain and function after total knee arthroplasty. Pain 2014; 155(10): 2040-6. [http://dx.doi.org/10.1016/j.pain.2014.07.007] [PMID: 25064836]

[37] Caracciolo B, Giaquinto S. Determinants of the subjective functional outcome of total joint arthroplasty. Arch Gerontol Geriatr 2005; 41(2): 169-76.

[http://dx.doi.org/10.1016/j.archger.2005.01.005] [PMID: 16085068]

[38] Lingard EA, Riddle DL. Impact of psychological distress on pain and function following knee arthroplasty. J Bone Joint Surg Am 2007; 89(6): 1161-9. [http://dx.doi.org/10.2106/JBJS.F.00914] [PMID: 17545417]

[39] Gandhi R, Razak F, Davey JR, Mahomed NN. Metabolic syndrome and the functional outcomes of hip and knee arthroplasty. J Rheumatol 2010; 37(9): 1917-22.

[http://dx.doi.org/10.3899/jrheum.091242] [PMID: 20634242]

[40] Clement ND, Jenkins PJ, MacDonald D, et al. Socioeconomic status affects the Oxford knee score and short-form 12 score following total knee replacement. Bone Joint J 2013; 95-B(1): 52-8. [http://dx.doi.org/10.1302/0301-620X.95B1.29749] [PMID: 23307673]

[41] Jones CA, Voaklander DC, Johnston DW, Suarez-Almazor ME. The effect of age on pain, function, and quality of life after total hip and knee arthroplasty. Arch Intern Med 2001; 161(3): 454-60. [http://dx.doi.org/10.1001/archinte.161.3.454] [PMID: 11176772]

[42] Ayers DC, Franklin PD, Ploutz-Snyder R, Boisvert CB. Total knee replacement outcome and coexisting physical and emotional illness. Clin Orthop Relat Res 2005; 440(440): 157-61.

[http://dx.doi.org/10.1097/01.blo.0000185447.43622.93] [PMID: 16239800]

[43] Carr JL, Moffett JA. The impact of social deprivation on chronic back pain outcomes. Chronic Illn 2005; 1(2): 121-9. [PMID: 17136918]

[44] Symmons DP. Environmental factors and the outcome of rheumatoid arthritis. Best Pract Res Clin Rheumatol 2003; 17(5): 717-27. [http://dx.doi.org/10.1016/S1521-6942(03)00063-9] [PMID: 12915154]

[45] Katz NP, Paillard FC, Ekman E. Determining the clinical importance of treatment benefits for interventions for painful orthopedic conditions. J Orthop Surg 2015; 10(1): 24 .

[http://dx.doi.org/10.1186/s13018-014-0144-x] [PMID: 25645576]

[46] Chesworth BM, Mahomed NN, Bourne RB, Davis AM. Willingness to go through surgery again validated the WOMAC clinically important difference from THR/TKR surgery. J Clin Epidemiol 2008; 61(9): 907-18. [http://dx.doi.org/10.1016/j.jclinepi.2007.10.014] [PMID: 18687289]

[47] Clement ND, MacDonald D, Simpson AH. The minimal clinically important difference in the Oxford knee score and Short Form 12 score after total knee arthroplasty. Knee Surg Sports Traumatol Arthrosc 2014; 22(8): 1933-9. [http://dx.doi.org/10.1007/s00167-013-2776-5] [PMID: 24253376]

[48] Dougados M. It's good to feel better but it's better to feel good. J Rheumatol 2005; 32(1): 1-2 [PMID: 15630716]

[49] Escobar A, Gonzalez M, Quintana JM, et al. Patient acceptable symptom state and OMERACT-OARSI set of responder criteria in joint replacement. Identification of cut-off values. Osteoarthritis Cartilage 2012; 20(2): 87-92. [http://dx.doi.org/10.1016/j.joca.2011.11.007] [PMID: 22155074]

[50] Escobar A, Quintana JM, Bilbao A, Aróstegui I, Lafuente I, Vidaurreta I. Responsiveness and clinically important differences for the WOMAC and SF-36 after total knee replacement. Osteoarthritis Cartilage 2007; 15(3): 273-80. [http://dx.doi.org/10.1016/j.joca.2006.09.001] [PMID: 17052924]

[51] Engel C, Hamilton NA, Potter PT, Zautra AJ. Impact of two types of expectancy on recovery from total knee replacement surgery (TKR) in adults with osteoarthritis. Behav Med 2004; 30(3): 113-23. [http://dx.doi.org/10.3200/BMED.30.3.113-123] [PMID: 15816314] 
[52] Smith BW, Zautra AJ. The role of purpose in life in recovery from knee surgery. Int J Behav Med 2004; 11(4): 197-202. [http://dx.doi.org/10.1207/s15327558ijbm1104_2] [PMID: 15657019]

(C) Lungu et al.; Licensee Bentham Open.

This is an open access article licensed under the terms of the Creative Commons Attribution-Non-Commercial 4.0 International Public License (CC BY-NC 4.0) (https://creativecommons.org/licenses/by-nc/4.0/legalcode), which permits unrestricted, non-commercial use, distribution and reproduction in any medium, provided the work is properly cited. 